\title{
Determining the Absolute Orientation in a Corridor Using Projective Geometry and Active Vision
}

\author{
Siniša Šegvić, Student member, IEEE, and Slobodan Ribarić, Member, IEEE \\ Department of Electronics, Microelectronics, Computer and Intelligent Systems \\ Faculty of Electrical engineering and computing \\ Unska 3, 10000 Zagreb, Croatia \\ fax (385) 016129 653, e-mail sinisa.segvic@fer.hr
}

\begin{abstract}
Parts of this work were previously published in proceedings of the IEEE International Symposium on Industrial Electronics, July 12-16, 1999, Bled, Slovenija, and in proceedings of the Electrotechnical and Computer Science Conference, September 23-25, 1999, Portorož, Slovenija.
\end{abstract}

\section{Abstract}

The capability of a mobile robot to determine its position in the environment (self-localization) is a prerequisite for achieving autonomous navigation. An approach is proposed for determining the absolute orientation of an autonomous robot in a system of corridors, based on the projective geometry and active computer vision. In the proposed approach, the common direction of longitudinal corridor edges is inferred by detecting the vanishing point of the corresponding straight line segments in the image. It is assumed that the knowledge about the vertical direction in the scene is available, so that the image coordinates of these vanishing points are considerably constrained. However, longitudinal corridor edges are not visible in images acquired for many viewing directions, so that the processing in a localization procedure has to be performed on a sequence of images acquired from the given position, for regularly arranged orientations of the camera. Extensive experimentation was performed on real scenes and the obtained results are provided.

\section{Keywords:}

Machine vision, active vision, robot vision systems, image recognition, image edge analysis, image line pattern analysis, orthogonal trihedral structure, projective geometry, vanishing points. 


\section{Introduction}

The field of autonomous navigation studies sensing and control strategies supporting the purposive movement of a physical mobile agent without human intervention. The capability of the agent to determine its position in the environment (self-localization) is the first and essential step towards achieving that objective. However, despite numerous approaches described in the literature, there is no satisfactory, truly elegant and flexible solution for the problem [Borenstein96]. This is particularly true if an unmodified environment is considered, i.e. an environment which was not specifically engineered in order to simplify the navigation. It seems that machine vision is potentially the most flexible and powerful source of information for such a task, specially in environments designed for humans who also use vision to support navigation.

The problem of vision based localization in unmodified indoor environments has recently been addressed by many researchers, due to to many potential applications of autonomous navigation in large buildings. Many of these applications involve intelligent mobile agents such as delivery robots for offices and factories [Kosaka95], robot guides at museums and exhibitions [Burgard98], domestic housekeeping robots, or maintenance robots in harmful or hazardous environments. Several years ago, a new promising technology of wearable computer devices appeared, allowing for new possibilities such as systems for assisting visually impaired persons and wearable personal computers capable of making context-aware decisions [Aoki99]. However, most of the known designs require that a model of the environment is known and use vision only to improve the accuracy of the position obtained by odometry, i.e. by measuring the displacement after the last accurate localization [Kosaka95, Burgard98].

A different approach was recently proposed in [Sim98] and [Davison98], in which many assumptions about the particular environment can be avoided. In the proposed designs, input images are searched for distinctive features, such as homogeneous rectangular patches with high gradient against 
the background. Self-location is consequently determined by triangulation, under assumption that most features can be recognized from many different positions in the environment. Despite the attractiveness, such approach could difficultly be applied in highly structured environments such as corridors where most of distinctive features are visible from only a small subset of all locations in the navigation area. Additionally, the possible set of moving directions in these environments is strongly constrained, so that it seems appropriate to design special navigation algorithms which would exploit such a property. A design following that approach was proposed by Schuster et al. [Schuster93] who described a procedure for steering a robot along the corridor by detecting rectangular elements of the ceiling structure. An another version of that approach was proposed by Dulimarta and Jain [Dulimarta97] who described an implementation of the complete navigation procedure based on the detection of ceiling lights and doorplate numbers.

We propose a procedure for determining the orientation of an autonomous robot in a generic corridor - an abstraction representing the most distinguished traits of real corridors including the orthogonal trihedral structure and the oblong shape. The former trait implies that object boundaries are flat surfaces having one of the three mutually orthogonal directions, while the latter stresses that one dimension of the environment is considerably larger than the others. A suitable referential coordinate system of the world in such an environment is the Cartesian coordinate system centered at a distinct point $\mathbf{O}$, with $\mathbf{X}, \mathbf{Y}$ and $\mathbf{Z}$ axes corresponding to transversal, longitudinal and vertical corridor directions, respectively, as shown in Fig.1(a). An important fact is that the knowledge about the vertical direction in the environment is implicitly available since the flat horizontal floor supports the frame of the robot. Alternatively, if the floor of the corridor is uneven, the vertical direction can be determined as the local orientation of the gravitational field, by a measurement device called dual-axis inclinometer or tilt sensor. These devices are sold as low cost "off-the-shelf" components, while their use is discussed in [Barshan95] and reported in [Matijevic98]. It has been shown that analogous devices are also "used" by many animals and that there are strong connections 
between the perceived vertical direction and the interpretation of the visual stimulus [Lebegue93]. Thus, vertical directions in coordinate systems of the world and the robot coincide, as depicted in Fig.1(a), while the full localization implies determining the origin of the robot centered coordinate system $\mathbf{o}$ and its orientation $\varphi$ in the world coordinates. It is assumed that the robot is equipped with a controllable camera with known intrinsic parameters [Mohr96]. The camera has two degrees of freedom: pan angle $\left(\gamma_{p}\right)$ and tilt angle $\left(\gamma_{t}\right)$ - Fig.1(b). These angles determine the orientation of the camera (viewing direction) in the robot coordinates. The camera is attached to the robot in such a way that, for pan and tilt angles of $0^{\circ}$, the optical axis coincides with the $\mathbf{y}$ axis of the robot centered coordinate system.

The proposed procedure is based on the analysis of a special perspective effect called vanishing point, and is performed in two stages. The first stage consists of detecting hypothetical vanishing points corresponding to horizontal corridor edges, in a sequence of images acquired from the given location, for a set of regularly arranged viewing directions. In the second stage, the obtained points are clustered in the coordinate system of the robot, and the strongest clusters are used for inferring directions of longitudinal corridor axes. The determined knowledge of the robot orientation can be used either for inferring the possible directions of advancement [Schuster93], or for enhancing further analysis of the scene [Shakunaga92, Lebegue93, Parodi96], or in correcting large lateral errors which are intrinsic to the odometry as the most widely used localization technique [Borenstein96]. Unlike previous approaches [Schuster93, Dulimarta97] who detect features which are present only in particular corridors, the procedure relies on long longitudinal edges as a feature present in all corridors. Consequently, we expect that our approach will be applicable in a broader class of real corridors. However, in order to obtain best results, a full-fledged industrial robot could enforce the results obtained by the proposed generic localization procedure with a number of specific procedures applicable to the particular corridor.

The following section describes the assumed model of image formation and discusses the use of 
vanishing points for interpreting perspective images of the considered environment in view of the obtained preprocessing results. Section 3 describes a method for detecting hypothetical vanishing points of the horizontal edges of the scene, while the proposed localization procedure is explained in section 4 . The results of extensive experimentation are provided.

\section{Assumptions}

\section{$2.1 \quad$ Projective geometry}

The image formation in an ideal camera can be modeled as a perspective projection of 3D space onto the image plane. Due to straightforward visualization, the image plane $\pi$ is often represented as Euclidian plane $\mathcal{R}^{2}$, as shown in Fig.2. However, in the context of 3D machine vision, a projective view of the image plane is more convenient since it allows for numerous simplifications such as describing the perspective transformation by linear equations and avoiding the possibility of singularities in formulas for manipulating points and lines [Mohr96]. Points of projective plane $\mathcal{P}^{2}$ are represented by $3 \mathrm{D}$ vectors $[X, Y, Z]^{\top}$ in which at least one coordinate is different from zero. These vectors are defined up to a constant which means that $[X, Y, Z]^{\top}$ and $[k \cdot X, k \cdot Y, k \cdot Z]^{\top}$ refer to the same point in $\mathcal{P}^{2}$, for each $k \neq 0$. There is a simple connection between 3D points in coordinate system of the camera (see Fig.2) and elements of $\mathcal{P}^{2}$, since the coordinates of each 3D point denote a vector referring to the element of $\mathcal{P}^{2}$ to which the given point maps to. In other words, the set of all vectors referring to an element of $\mathcal{P}^{2}$ corresponds to a 3D line which consists of all points mapping to that element. The points $[X, Y, 0]^{\top} \in \mathcal{P}^{2}, X \neq 0 \vee Y \neq 0$ are called ideal points since they arise as intersections of parallel lines in $\mathcal{P}^{2}$. Consequently, they do not have an interpretation in $\mathcal{R}^{2}$ and the projective plane can be thought of as a union of ideal points and the usual Euclidian plane $\mathcal{R}^{2}$. 
The isomorphical mapping from $\mathcal{R}^{2}$ to $\mathcal{P}^{2}$ is described by the standard injection:

$$
(x, y) \mapsto[x, y, 1]^{\top}
$$

while the inverse mapping is given by:

$$
[X, Y, Z]^{\top} \mapsto\left(\frac{X}{Z}, \frac{Y}{Z}\right), Z \neq 0
$$

A fundamental property of $\mathcal{P}^{2}$ is that there exists a duality between points and lines such that for every statement there exists a corresponding dual statement in which the roles of points and lines are interchanged. The basic relations between points and lines in $\mathcal{P}^{2}$ are described by the following equations [Kanatani91]:

A line $\mathbf{l}$ consists of points $\mathbf{p}$ satisfying the equation ${ }^{1}$ :

$$
(\mathbf{l}, \mathbf{p})=0
$$

A line $\mathbf{l}$ intersects points $\mathbf{p}_{\mathbf{1}}$ and $\mathbf{p}_{\mathbf{2}}$ iff:

$$
l=p_{1} \times p_{2}
$$

Two lines $\mathbf{l}_{\mathbf{1}}$ and $\mathbf{l}_{\mathbf{2}}$ intersect in the point $\mathbf{p}$ iff:

$$
\mathrm{p}=\mathrm{l}_{1} \times \mathrm{l}_{2}
$$

\subsection{Vanishing points}

It is well known that perspective projection maps each set of parallel lines from 3D space into the set of half lines in the projective plane with a common endpoint called a vanishing point [Shakunaga92, Schuster93, Lutton94, McLean95, Shufelt99]. There is a one-to-one correspondence between the points of the projective plane (vanishing points) and directions (sets of parallel lines) in 3D space. Thus, finding vanishing points which are associated with main directions in the scene becomes an

\footnotetext{
${ }^{1}(\mathbf{a}, \mathbf{b})$ and $\mathbf{a} \times \mathbf{b}$ denote scalar and vector products of vectors $\mathbf{a}$ and $\mathbf{b}$, respectively.
} 
attractive goal because it allows drawing conclusions about its 3D structure. Similar considerations apply to sets of parallel planes which, in a perspective image of the scene, appear as converging to the vanishing line corresponding to their $3 \mathrm{D}$ orientation. The position of vanishing points corresponding to lines which are situated on a certain plane is constrained to its vanishing line and each point of the vanishing line is a vanishing point for one set of parallel lines in that plane.

In an image of a scene with orthogonal trihedral structure, the set of all extracted lines can be partitioned into three subsets so that lines from each subset correspond to a different subset of parallel edges in the scene and intersect the associated vanishing point [Lebegue93]. The locations of the three vanishing points in these scenes are mutually dependent. Particularly, if the vertical direction in the scene is known (see Fig.3), the corresponding vanishing point $\mathrm{VP}_{\mathrm{V}}$ can be determined directly, together with the horizon which is the vanishing line for horizontal planes of the scene. The remaining two vanishing points, $\mathrm{VP}_{\mathrm{H} 1}$ and $\mathrm{VP}_{\mathrm{H} 2}$ in Fig.3, are situated on the horizon and are still mutually dependent.

\subsection{Generic corridor scenes}

An intrinsic property of a generic corridor is that its length is considerably greater than its width and its height. The analyzed class of generic corridor scenes is therefore described as the subset of orthogonal trihedral scenes in which longitudinal edges are considerably longer than all other edges and, in particular, more numerous than transversal ones. For many viewing directions, it can therefore be expected that line segments corresponding to longitudinal edges should also be more numerous than the segments corresponding to transversal edges. In images obtained for these viewing directions, the vanishing point for longitudinal directions of the scene can be determined as the dominant vanishing point of all horizontal directions, i.e. the vanishing point determined by the greatest number of most unambiguous line segments (see Fig.3). 


\subsection{Preprocessing}

Because of the regular structure of the considered environment, each acquired image is described in terms of straight line segments before further processing. The chosen representation is a tradeoff between the capability of reliable description and the possibility of devising efficient algorithms. Line drawing is one of the most common ways of human communication (e.g. technical drawings, comics) so that its expressive power is probably more than sufficient. On the other hand, the count of individual straight lines in images of the considered environment is often less than the count of pixels in source images for several orders of magnitude (typically, 50 vs. 50000). Therefore, algorithms for processing line drawings are regularly less complex than algorithms for image processing.

The description of the original image in terms of weighted line segments is obtained in two stages: edge detection and edge linking. The employed edge detection algorithm is a modification of the known Canny edge detector [Canny83, Heath96], in which each edge element is assigned a weight equal to the local intensity of the gradient in the image. Line segments are extracted by an iterative detection of collinear edge elements based on the modified Hough Transform [Illingworth88, Lie90]. The weight of each segment is calculated on the basis of its length and the sum of weights of the constituting edge elements. An attempt has been made to design these procedures in a flexible manner so that many parameters (e.g. thresholds in edge detection, for detecting local maxima in parameter space, for filtering the extracted line segments etc.) are dynamically determined by heuristic algorithms, depending on statistical properties of the input. Nevertheless, the subjective quality of the technique strongly depends on the image being analyzed. Best results are obtained on images of scenes with controlled illumination with few straight edges which are relatively long compared to image dimensions. Unfortunately, the preprocessing results obtained on scenes with uncontrolled illumination does not guarantee the success in applying the known object recognition techniques. Real corridor scenes tend to be illuminated "sufficiently" from the human point of 
view but badly and unevenly in the context of image acquisition. Moreover, the navigation implies activity over considerable space and time so that it can not be expected that the conditions for image acquisition will be uniform, particularly in unknown environments. On the other hand, human interventions such as specifying parameters for edge detection or telling the robot where to look next are not acceptable so that it may seem that, at present, machine vision can hardly be used in uncontrolled environments.

Lebegue and Aggarwal [Lebegue93] however state that the results of techniques for retrieving the description of the image in terms of line segments can substantially improve if the 3D directions of corresponding edges are known. Parodi and Piccioli [Parodi96] have assumed that the locations of vanishing points corresponding to main structural axes of the scene are known à priori and thus simplified the problem of the 3D reconstruction of the scene. Shakunaga [Shakunaga92] argues that a "general" approach to object recognition is not adequate when the appearance of the object strongly depends on the viewing direction. He therefore proposes the determining of an object centered coordinate system as the first step in the analysis of an indoor scene.

\section{The detection of vanishing points}

In many applications of machine vision, the internal parameters of the camera are known so that 3D direction of parallel edges in the scene can be determined from the vanishing point of the corresponding line segments in the image [Shufelt99]. Such inferring of the 3D structure is particularly convenient in environments with many parallel edges having relatively few 3D directions. Indoor scenes are often good representatives of such an environment [McLean95], but also urban outdoor scenes [Lutton94] and scenes associated with automated steering of vehicles in urban [Parodi96] and extra-urban roads [Liou87]. The achieved knowledge about the main structural directions of the scene can substantively improve the conditions for further understanding of the scene [Shakunaga92, Lebegue93, Parodi96]. 


\subsection{Approaches to the detection of vanishing points}

The key assumption in the context of detection of vanishing points in perspective images is that groups of parallel edges in the scene correspond to the most numerous groups of converging line segments in the projective plane. The known methods can be classified in two basic approaches regarding the required preprocessing procedure.

A method for detecting vanishing points which performs processing directly on the set of edge elements was applied to the task of automated vehicle steering [Liou87]. In that work, it was assumed that the road is locally flat and straight (which allows determining the vanishing line corresponding to the road plane) and that the vanishing point is situated within image boundaries. These assumptions allowed the reduction of the search space for the vanishing point corresponding to the road boundaries from the whole image plane to a single line segment in the image. The obtained search space is partitioned into short sectors and a voting process on the set of edge elements of the analyzed image is used to find the sector which most likely contains the vanishing point.

However, most methods assume that a description of the analyzed image in terms of line segments has already been determined by a generic preprocessing procedure. These methods try to find groups of line segments intersecting the common point of the projective plane and can be further classified with respect to the clustering approach.

\subsubsection{Clustering based on the Hough Transform}

In this approach, a Hough transform (HT) is applied to the set of extracted line segments in order to obtain its representation in parameter space which would hopefully allow for easier detection of vanishing points. Each point of the parameter space should represent one vanishing point or, equivalently, one direction in 3D space. A good choice for parameter space, therefore, is the unit sphere centered at the origin (Gaussian sphere) since it is finite and complete. The approach implies forming the adequate partition of the parameter space and assigning an unique accumulator to each 
member of that partition. Consequently, contributions of single line segments [Lutton94, Shufelt99] (or of pairs of line segments [Schuster93]) to relevant accumulators are determined. The approximate positions of vanishing points are finally detected by searching for local maxima on the set of accumulators. This approach is used by most of the existing methods since it formalizes the clustering process.

\subsubsection{Explicit clustering}

In the other clustering approach, for each pair of line segments a hypothesis is made that it corresponds to a pair of parallel edges in the scene. The resulting hypothetical vanishing points are subjected to an explicit clustering procedure, either on their projections to the Gaussian sphere [Shakunaga92], or in the image plane itself [McLean95]. The centers of the obtained clusters are finally proclaimed as probable vanishing points. While the classic methods for detecting vanishing points using the HT offer a sound general approach, they may not be the best option for each particular task. McLean and Kotturi [McLean95] emphasize that the difficulties in finding local maxima in parameter space lead to a procedure which requires à priori knowledge about their approximate position. They further argue that the accuracy of obtained results is limited by the resolution of the parameter space and may not achieve the limit imposed by the accuracy of the input data.

\subsection{The proposed method}

Based on specific properties of the considered environment, while taking into account the results of previous research, we chose an approach to the detection of vanishing points based on explicit clustering. On the first sight, such approach does not seem to be attractive since the purpose of the HT in the first place is to formalize the clustering procedure and reduce its complexity. Nevertheless, explicit clustering can be adequate when the count and locations of vanishing points are heavily constrained, which is exactly the case in images of the considered environment. The fact that the 
knowledge about the vertical direction in the scene is available additionally simplifies the task, since it makes it possible to determine the vanishing point for vertical edges in the scene [Lebegue93] and the horizon [Liou87] directly. These constraints can diminish the count of hypothetical vanishing points for several orders of magnitude so that the complexity of the clustering stage may become neglectable.

On input, the proposed method requires the list of weighted line segments extracted from the analyzed image. On output, the method produces a list of weighted clusters of vanishing points for horizontal directions in the scene. The dominant cluster (i.e. the cluster with the greatest weight) is expected to reflect the vanishing point corresponding to the longitudinal direction. The method is performed in four stages which are described in the following paragraphs.

\subsubsection{Electing consistent line segments}

In this stage, line segments for further consideration are selected from the set obtained in the preprocessing stage. Line segments which intersect the horizon are discarded first, since they can not correspond to horizontal edges in the scene and are not relevant in this context. Short line segments have an inherent large direction uncertainty [Shufelt99]. Fortunately, all object boundaries in the considered environment are straight edges so that the analysis can be limited only to relatively long line segments. Thus, line segments which are shorter than $1 / 20$ of the shorter dimension of the image are also discarded (the factor 1/20 was determined experimentally). Finally, it is also possible to discard line segments intersecting the vanishing point for the vertical direction in the scene, since it is very likely that they correspond to vertical edges. This operation significantly simplifies further processing, since, in images of the considered environment obtained for prevalently horizontal viewing directions, most of extracted line segments correspond to vertical edges. 


\subsubsection{Determining homogeneous line equations}

For each elected line segment, the homogeneous line equation (3) is determined in two steps. First, the homogeneous representations of the two endpoints of a line segment are determined by injection (1). Then, the homogeneous equation of the line passing through the obtained endpoints is determined, according to (4).

\subsubsection{Determining hypothetical vanishing points}

For each pair of elected line segments, a hypothetical vanishing point is calculated according to (5), under the assumption that the corresponding edges are parallel and horizontal — Fig.4. If the position of the hypothetical vanishing point is inside one of segments, line segments can not correspond to parallel edges and the point is discarded. Moreover, the point is also discarded if it is considerably distant from the horizon, since that also contradicts the assumption. Each remaining hypothetical vanishing point is assigned the weight equal to the product of weights of the corresponding segments.

\subsubsection{Clustering of hypothetical vanishing points}

The clustering procedure is performed on the Gaussian sphere since the chosen metric (the squared Euclidian distance) is more uniform on the sphere than in the projective plane. Hypothetical vanishing points from the obtained set are therefore projected to the sphere and are subsequently subjected to the unsupervised clustering using the "maximin" algorithm [Tou74]. Each obtained cluster is assigned the weight being equal to the sum of weights of its elements. Finally, the centroid of the cluster with the greatest weight is proclaimed as the dominant vanishing point for horizontal directions in the scene. 


\subsection{Experimental results}

The described method for detecting vanishing points was tested both on real corridor images and on sets of generated pseudorandom line segments. Real images were acquired under both day-time and night-time conditions, for different orientations of the camera. The primary objective was to test the correct functionality of the method on images containing many longitudinal edges. However, correct behavior of the method was also tested on images not satisfying the requirements for a successful determination of the longitudinal vanishing point.

\subsubsection{A preliminary experiment}

An example of an image containing enough information for a correct determination of the longitudinal vanishing point is shown in Fig.5(a). Fig.5(b) shows the description of the acquired image in terms of straight line segments, determined by the preprocessing procedure. The width of segments designates their weights while an additional dashed line over a segment indicates that it was elected as a segment which might correspond to a longitudinal corridor edge (see the previous subsection). The results are shown in Fig.5(c) as the representations of hypothetical vanishing points (crosses) and of centroids of obtained clusters (squares) in Euclidian plane $\mathcal{R}^{2}$. Fig.5(c) also shows the segments which support the dominant cluster (thick lines), other elected segments (dashed lines), and the horizon (dashed horizontal line). The quantitative processing summary is shown in Fig.5(d) as the set of relevant camera parameters, counts of line segments at various processing stages, properties of the dominant cluster, as well as the timing profile. The camera parameters include pan and tilt angles (see Fig.1), together with horizontal and vertical fields of view, measured in degrees. The properties of the dominant cluster include the Euclidian coordinates of its centroid, the corresponding direction of the longitudinal corridor axis as the final processing result, the count of hypothetical vanishing points, and finally the weight of the cluster. The timing profile shows the execution times of the following execution stages: Gaussian smoothing, the rest of Canny edge detection, the procedure for finding 
lines and finally the procedure for detecting vanishing points which regularly took less than the system timing resolution. The experiment was performed on a computer with the integer performance of 9.8 SPECint95, while the dimension of the input image were $320 \times 240$ pixels.

The set of elected line segments shown in Fig.5(b) includes two segments which do not reflect the structure of the environment. These segments are caused by a combination of shadows and reflections on the corridor floor due to the excessive illumination through the fire-exit door. The first of the two segments is located near the horizon and is directed towards the neighborhood of the dominant cluster. Consequently, it participates in the process of determining the cluster centroid and therefore increases the uncertainty of the output. The other segment is located in the bottom of the image and is directed far enough from the dominant cluster. The resulting two hypothetical vanishing points are assigned to a separate cluster which is later rejected since its weight is much smaller than the weight of the dominant cluster.

\subsubsection{The reliability of the method}

Despite the significant design effort, the preprocessing stage turned out to be the limiting factor for the reliability of the proposed method. The position and, particularly, the direction of detected line segments sometimes quite differed from the projection of the corresponding edges to the image plane. This effect is mainly due to the statistical nature of the Hough transform which makes a quantitative error analysis extremely difficult [Palmer97]. The effect is particularly strong for short image segments but can also affect the longer ones, particularly in the vicinity of an illumination discontinuity — e.g. the larger segment corresponding to the lower-left longitudinal corridor edge in Fig.5(c). Additionally, experiments shown that the segments with greatest weights regularly correspond to vertical edges of the scene. Consequently, in some positions of the corridor, the preprocessing stage did not detect any useful line segments in the acquired image and the method produced no results. As designed, the preprocessing stage never reported a false positive result i.e. a 
line segment not corresponding to a significant straight discontinuity of pixel intensity in the image. However, in few cases of extremely bad illumination, reflexes and shadows were more distinct than discontinuities caused by longitudinal edges and the method produced wrong results.

The results obtained for non-zero tilt angles of the camera were significantly better than the results for horizontal viewing directions since longitudinal edges appear as larger line segments if the viewing direction is somewhat inclined (see Fig.6). The best choice for the tilt angle would be near the half of the vertical field of view of the camera since it ensures near-maximal visible lengths of longitudinal corridor edges, for all combinations of corridor widths and widths of the field of view of the camera. The method performed better for viewing directions inclined upward (i.e. for positive tilt angles) than for downward inclined ones, since the former regularly include more longitudinal edges due to doors and windows, while the latter always contain effects of the highly reflective marble floor which increase the possibility of recognition errors. Unfortunately, the inclined viewing direction implies that upper and lower longitudinal corridor edges are often not both detected by the preprocessing procedure, which increases the uncertainty of the results obtained for images containing only one corridor wall. In such images, all longitudinal edges are relatively close and therefore nearly parallel, as shown in Fig.7. Consequently, the calculated positions of hypothetical vanishing points are much more sensitive to directional errors of extracted line segments than for views containing distant longitudinal edges, which is reflected by a larger dispersion of points of the dominant cluster. For the same reasons as before, the described effect has larger influence in images obtained for viewing directions inclined downward (see Fig.7). The influence of the effect can be modeled by an additional adjustment of the weight of hypothetical vanishing points, depending on the intersection angle of the corresponding segments. 


\subsubsection{The results of comprehensive experimentation}

The results for a total of 132 experiments performed at 6 different corridor locations, for 22 different viewing directions (2 tilt and 11 pan angles of the camera) are summarized in Table 1. Rows of the table correspond to individual experiments performed for different pan angles relative to the longitudinal corridor axis (column $\gamma_{p}$ ), at different corridor locations (column \#). For each experiment, the three columns labeled count of line segments contain the total count of extracted line segments, the count of elected segments and the count of segments supporting the dominant cluster. The last two columns contain the weight of the dominant cluster and the deviation $\delta$ of the determined longitudinal corridor direction from the ground truth. Mutual dependencies of the data from Table 1 are illustrated in Fig.8, in which the average relative weight and the average deviation $\delta$ obtained in locations from $\mathrm{A}$ to $\mathrm{F}$ are plotted against the pan angle of the camera, for both tilt angles $\gamma_{t}$. The average weights obtained for $\gamma_{t}=15^{\circ}$ were significantly stronger than for $\gamma_{t}=-15^{\circ}$, which confirms observations from the paragraph 3.3.2. The obtained results indicate that the proposed method can be used for reliable determining of the longitudinal corridor direction for viewing directions differing from the longitudinal for less than $30^{\circ}$, which is mainly determined by the horizontal field of view of the used camera $\left(47^{\circ}\right)$. The results also show that the method rarely gives false positive answers and that the accuracy of the obtained results (deviation $\delta$ ) is strongly coupled with the weight of the dominant cluster. Finally, the results illustrate the importance of choosing the "right" viewing direction in analyzing corridor scenes since, for many viewing directions, the acquired images do not contain useful information for navigational purposes (pan angles of $45^{\circ}, 60^{\circ}$ and $\left.90^{\circ}\right)$. An active vision approach for determining useful viewing directions is therefore proposed in the following section. 


\subsubsection{Results for pseudo-random line segments}

Finally, we tested the possibility of detecting the low consistency of the results by analyzing the ratio between the count of segments supporting the dominant cluster and the count of elected segments. Experiments were performed in three series, for different counts of generated pseudo-random line segments and the obtained results are summarized in rows of Table 2. For each series, the count of performed experiments is listed in column 2, while columns 3 through 5 contain the total count of generated line segments, and average counts of the elected segments and of the segments supporting the dominant cluster. Finally, columns 6 and 7 contain the average ratio between the count of supporting line segments and the count of elected segments, and the corresponding standard deviation for all performed experiment in a series. Experiments shown that the mentioned ratio is significantly and consistently lower for sets of pseudo-random segments than in real images (see Table 1). That ratio can therefore be used as an empirical evidence that the obtained results are not accidental, i.e. that they actually reflect the structure of the environment.

\section{The localization procedure}

This section describes an application of the method for the detection of vanishing points of the horizontal corridor edges in a localization procedure. The objective of the designed procedure is to determine the orientation of the observer in an unknown corridor. The procedure is conceived as a basic navigational routine which is applied in situations in which no applicable knowledge about the environment is available. Such exceptional situations include a "wake up" procedure [Krotkov89] after periodic maintenance of the mobile robot [Sim98], entering the unknown environment (e.g. after leaving the elevator) and the occurrence of contradictory conclusions in control procedures at hierarchically higher levels [Dulimarta97]. In that context, the robustness of the localization procedure is considered more important than the execution speed. 


\subsection{The proposed approach}

The basic assumption for success of any method for detecting vanishing points is that the analyzed image contains many parallel edges. However, in images of the considered environment, that assumption holds only if the viewing direction is close to the longitudinal corridor axis, depending on the horizontal field of view of the utilized camera. Consequently, the results of a localization procedure based on detecting vanishing points in a single image of the environment, could be relied upon only if the approximate orientation of the robot is already known. But that would be contrary to the objectives set at the beginning of the section — the desired procedure should be applied exactly in those exceptional situations when all other sources of knowledge are unavailable or uncertain. It seems that the only possible way of solving the problem would be to apply principles of active perception and to bring decisions based on analyzing the whole sequence of images, acquired for several viewing directions. The results obtained by the method described in the previous section (Tables 1 and 2), suggest analyzing a sequence of images, obtained for different pan angles of the camera.

\subsection{Implementation details}

The proposed implementation is subdivided in two processing stages. The first stage is applied to each image of the sequence acquired from the given location for the set of regularly arranged viewing directions. The stage consists of detecting hypothetical vanishing points corresponding to horizontal directions of the scene and transforming them to the coordinate system of the robot. The collected points are clustered in the second stage, and centroids of the obtained clusters are used for determining the directions of longitudinal corridor axes in the coordinate system of the robot.

The analyzed images are acquired for pan angles of the camera $\gamma_{p}=k \cdot \Delta \gamma_{p}, k=0,1, \ldots, n_{\text {views }}-1$, where $\Delta \gamma_{p}=2 \pi / n_{\text {views }}$. The value of $n_{\text {views }}$ thus determines $\Delta \gamma_{p}$ and is chosen on the basis of the

results listed in Table 1. $\Delta \gamma_{p}$ must be small enough in order to ensure the acquisition of several 
images containing enough information for a correct determination of the longitudinal vanishing point. However, it should not be too large in order to ensure maximal execution speed for a given level of robustness. We therefore chose to set the $\Delta \gamma_{p}$ near the $1 / 3$ of the horizontal field of view of the camera. In the performed experiments the horizontal field of view was $47^{\circ}$, so that $n_{\text {views }}$ turned out to be 26 which determined $\Delta \gamma_{p}$ to be $13.85^{\circ}$. Following the discussion in the subsection 3.3 , the tilt angle for the procedure was set to $15^{\circ}$, near the half of the vertical field of view of the utilized camera. Additionally, the results from Table 1 indicate that the relative uncertainty of the obtained hypothetical vanishing points is considerably larger for points differing from the viewing directions for more than a half of the horizontal field of view. These points are consequently discarded and are not considered in the clustering stage, in order to improve the robustness of the procedure.

Following the above considerations, the $k$-th iteration of the first stage consists of the following operations:

\section{Image acquisition}

Pan and tilt angles of the camera are set to the following values: $\gamma_{p}=k \cdot \Delta \gamma_{p}$ and $\gamma_{t}=15^{\circ}$. An image is acquired with dimensions of $320 \times 240$ pixels.

\section{Preprocessing}

The acquired image is represented by the set of weighted straight line segments.

\section{Detection of hypothetical vanishing points}

The extracted set of weighted straight line segments is searched for hypothetical vanishing points, as described in subsection 3.2. The points which are close to the viewing direction are consequently transformed (Fig.9) to the coordinate system of the robot $(\varphi, \theta)$ and finally preserved for further processing in the second stage.

The robot centered coordinate system $(\varphi, \theta)$ is situated at the surface of the right cone whose slope and the main axis match the tilt angle of the camera and the vertical direction in the scene, respectively. 
The origin of the coordinate system is defined by the intersection of the surface of the cone with the optical axis of the camera for pan and tilt angles of $0^{\circ}$. The relations between the two coordinate systems are shown in Fig.9.

In the second stage, clusters of hypothetical vanishing points are formed using the "maximin" algorithm which was mentioned in subsection 3.2. Clusters with weights less than $0.01 \%$ relative to the weight of the dominant cluster are rejected as uncertain and probably related to illumination effects. Feasible directions of advancement are in the end determined on the basis of centroids of the resulting clusters.

\subsection{Experimental results}

The experiments were performed at 18 locations of the corridor at our department, as illustrated in Fig.10. The computer on which the processing was performed was mounted on a moving cart, and the camera shown in Fig.1(b) was placed at its most exposed position. The obtained results are summarized in Table 3. Rows of the table correspond to corridor locations a through $\mathbf{r}$ (Fig.10), together with matching orientations of the robot coordinate system $\varphi_{0}$. The table lists the following data about the detected clusters of hypothetical vanishing points: the coordinate $\varphi$ of the cluster in the robot centered coordinate system (positive $\varphi$ corresponds to the clockwise turn), the relative weight of the cluster $(w t)$ with respect to the strongest cluster, and the error calculated by comparing the centroid of the cluster to the ground truth $(\delta)$. The results illustrate that the procedure can be used for an accurate determination of longitudinal corridor directions. The experiments performed at locations $\mathbf{a}, \mathbf{g}, \mathbf{l}$ and $\mathbf{m}$, which are close to a corridor end, show the main limitation of the procedure — that the corridor is detected only if it extends at least 4 meters away from the observer.

The performance of the utilized computer was about 2 SPECint95, so that the processing of single images last $4 \mathrm{~s}$, and the total computation time was $104 \mathrm{~s}$. The time spent in procedures for controlling the camera was 48 s so that the total execution time of the procedure (response time) was 
about 2 minutes and a half. However, the computers with more than 40 SPECint95 are commonly found today so that the computation time on a recent computer would probably be around $5 \mathrm{~s}$ and is probably going to become neglectable in the near future. Such a dramatic improvement in the construction of the step motors is unlikely so that we expect that the industrial performance of the procedure would be bound by the mechanical capabilities of the camera drive.

Graphical representation of the results for the three characteristic locations in Fig.10 is shown in Fig.11. For the sake of the representation simplicity, points of the coordinate system of the robot $(\varphi, \theta)$ are transformed to the polar coordinate system $(r, \varphi)$. Upright direction in the polar coordinate system, corresponds to the angle of $0^{\circ}$ on the surface of the cone. The radius $r$ in the polar coordinate system is proportional to the coordinate $\theta$ on the surface of the cone, and is calculated as $r=r_{H}+\frac{\theta-\gamma_{t}}{k}$, where variables $r_{H}$ and $k$ are empirically determined constants. Points of the robot coordinate system which are situated on the horizon $\theta=0^{\circ}$, map to points of the polar plane with radius $r=r_{H}$. For each of the three experiments, two images of the resulting polar plane $(r, \varphi)$ after the transformation are shown. As in the previous section, crosses and squares correspond to detected hypothetical vanishing points and cluster centroids, respectively. Left images don’t make the right impression about the processing results since the weight of hypothetical vanishing points which are far from cluster centroids is usually considerably lower than the average. For that reason, right images are formed in the following four steps, as an alternative illustration of the processing results:

i) the values of all image pixels are initialized to 0 ;

ii) the weight of each hypothetical vanishing point is added to the value of the corresponding pixel;

iii) image is smoothed by a 2D Gaussian kernel with a large value of the parameter $\sigma$, and a circle corresponding to the horizon is drawn;

iv) arrows indicating the heading of the robot and the orientation of the world coordinate system 
are designated, together with the sketch of the corridor at the corresponding location.

Thus, the right image in Fig.11(a) indicates that there are three possible directions of advancement corresponding to the three corridors extending from the location $\mathbf{f}$ in Fig.10. Similarly, Fig.11(b) reflects that the robot is situated near the dead end of the corridor (location l), while Fig.11(c) indicates that the robot is in the middle of a simple corridor, far from corridor ends (location $\mathbf{n}$ ).

\section{Summary and conclusion}

In this paper, we described a method for detecting vanishing points corresponding to horizontal edges, which exploits the knowledge of the vertical direction in the scene. We have also shown the application of that method in a localization procedure based on an active vision approach. The proposed procedure determines the orientation of a mobile robot in a system of corridors. A straightforward approach to localization would be to apply the known object recognition methods and to match the perceived objects (landmarks) with the map of the environment. However, the experiences from the previous research and the results of our preliminary experimentation have shown that these methods perform well only for images obtained in controlled conditions (illumination, camera position and orientation). Such performance is not surprising since experiments have shown that the mentioned conditions strongly influence the quantity of useful information in acquired images. We therefore suggest an incremental approach to the understanding of the scene, in which each new processing step is triggered by the possibility of robust acquisition of further knowledge about the world, which can be useful in solving the high level navigation task. In accordance with the principles of active vision, it is favorable to equally specify both procedures for acquiring and for the processing of images. The processing steps in such a system must be simple and robust in order to support stable and secure navigation. The entire proceeding of such navigation can be seen as a great localization procedure since the robot continuously updates the knowledge about the world. Nevertheless, the 
term "localization" usually refers to the basic procedure which can be applied in uncertain situations in which prior experiences with the environment either do not exist or can not be trusted. The analysis of longitudinal corridor axes is adequate in such situations, since it is the most distinguished property of the considered environment.

The described approach to localization is similar to actions performed by people, while looking for a certain office in an unknown corridor. When a human enters the corridor (e.g. from an elevator), before performing any other actions, he or she must look around and find out the directions in which the corridor extends. Likewise, the proposed localization procedure, controls pan and tilt angles of the camera in order to acquire a sequence of images for a set of evenly arranged viewing directions. The final decision of the localization procedure is brought after detecting hypothetical vanishing points in each image of the sequence, by explicit clustering in the coordinate system of the robot. The obtained experimental results confirmed that the approach can provide reliable information in regular conditions, i.e. without the need for introducing additional illumination sources into the environment.

The presented work is a part of the research project aimed at improving the capability of visually impaired persons to move through indoor environments of public buildings. The project involves a synergy of several branches of computer science with the following wearable computer devices: a palm-top computer capable for voice synthesis and image acquisition, an ear-mounted headphone and the miniaturized camera attached to a lapel or mounted on a cap of the impaired person, supplemented with a tilt sensor. The future work will be directed towards techniques which will use and extend the obtained knowledge and, in cases of ambiguous or uncertain results, fall back to the basic procedure. Potential objectives in that context include the determination of physical dimensions of the corridor (i.e. width, height), distance between the robot and lateral walls and recognizing doors and door plates. 


\section{References}

[Aoki99] Hisashi Aoki, Bernt Schiele and Alex Pentland, Realtime personal positioning system for wearable computers, in Proceedings of the 3rd IEEE International Symposium on Wearable Computers, San Francisco, USA, October 1999.

[Barshan95] Billur Barshan and Hugh F. Durrant-Whyte, Inertial navigation systems for mobile robots, IEEE Transactions on Robotics and Automation, vol. 11(3), June 1995.

[Borenstein96] J. Borenstein, H. R. Everett and L. Feng, Navigating Mobile Robots: Sensors and Techniques, A. K. Peters, Ltd., Wellesley, MA, January 1996.

[Burgard98] W. Burgard et al., The interactive museum tour-guide robot, in Proceedings of the Fifteenth National Conference on Artificial Intelligence, Madison, Wisconsin, 1998.

[Canny83] John Canny, Finding edges and lines in images, Tech. Rep., Laboratory of Artificial Intelligence, Massachussets Institute of Technology, Cambridge, USA, June 1983.

[Davison98] A.J. Davison and D.W. Murray, Mobile robot localisation using active vision, in Proceedings of the 5th European Conference on Computer Vision, vol. II, pp. 809-825, Freiburg, Germany, June 1998.

[Dulimarta97] H.S. Dulimarta and A.K. Jain, Mobile robot localization in indoor environment, Pattern Recognition, vol. 30(1):99-111, January 1997.

[Heath96] Mike D. Heath, A robust visual method for assessing the relative performance of edge detection algorithms, Master's thesis, College of Engineering, University of South Florida, Department of Computer Science and Engineering, December 1996.

[Illingworth88] J. Illingworth and J.V. Kittler, A survey of the hough transform, Computer Vision Graphics and Image Processing, vol. 44(1):87-116, October 1988.

[Kanatani91] K.I. Kanatani, Computational projective geometry, Computer Vision Graphics and Image Processing - Image Understanding, vol. 54(3):333-348, November 1991.

[Kosaka95] Akio Kosaka and Juiyao Pan, Purdue experiments in model-based vision for hallway navigation, in Proceedings of Workshop on Vision for Robots, IROS'95 Conference, Pittsburgh, PA, 1995.

[Krotkov89] Eric P. Krotkov, Active computer vision by cooperative focus and stereo, Springer-Verlag, 1989.

[Lebegue93] X. Lebegue and J.K. Aggarwal, Significant line segments for an indoor mobile robot, IEEE Transactions on Robotics and Automation, vol. 9(6):801-815, December 1993.

[Lie90] Weng-Nung Lie and Yung-Chang Chen, Robust line-drawing extraction for polyhedra using weighted polarized hough transform, Pattern Recognition, vol. 23(3/4):261-274, 1990.

[Liou87] S.P. Liou and R.C. Jain, Road following using vanishing points, Computer Vision Graphics and Image Processing, vol. 39(1):116-130, July 1987.

[Lutton94] E. Lutton, H. Maitre and J. Lopez-Krahe, Contribution to the determination of vanishing points using hough transform, IEEE Transactions on Pattern recognition and Machine Intelligence, vol. 16(4):430-438, April 1994.

[Matijevic98] Jacob Matijevic, Autonomous navigation and the sojourner microrover, Science, vol. 280(5362):454-455, April 1998. 
[McLean95] G.F. McLean and D. Kotturi, Vanishing point detection by line clustering, IEEE Transactions on Pattern recognition and Machine Intelligence, vol. 17(11):1090-1095, November 1995.

[Mohr96] Roger Mohr and Bill Triggs, Projective geometry for image analysis, July 1996, URL http://www.inrialpes.fr/movi/people/Triggs/isprs96/isprs96.html, a tutorial given at the International Symposium of Photogrammetry and Remote Sensing, Vienna.

[Palmer97] P. L. Palmer, J. Kittler and M. Petrou, An optimizing line finder using a hough transform algorithm, Computer Vision Graphics and Image Processing - Image Understanding, vol. 67(1):1-23, July 1997.

[Parodi96] Pietro Parodi and Giulia Piccioli, 3d shape reconstruction by using vanishing points, IEEE Transactions on Pattern recognition and Machine Intelligence, vol. 18(2):211-217, 1996.

[Schuster93] R. Schuster, N. Ansari and A. Bani-Hashemi, Steering a robot with vanishing points, IEEE Transactions on Robotics and Automation, vol. 9(4):491-498, April 1993.

[Shakunaga92] Takeshi Shakunaga, 3-d corridor scene modeling from a single view under natural lighting conditions, IEEE Transactions on Pattern recognition and Machine Intelligence, vol. 14(2):293-298, February 1992.

[Shufelt99] Jefferey Shufelt, Performance evaluation and analysis of vanishing point detection techniques, IEEE Transactions on Pattern recognition and Machine Intelligence, vol. 21(3):282288, March 1999.

[Sim98] Robert Sim, Mobile robot localisation using learned landmarks, Master's thesis, McGill University, Montreal, Department of Computer Science, July 1998.

[Tou74] J.T. Tou and R.C. Gonzalez, Pattern Recognition Principles, Addison-Wesley, 1974. 


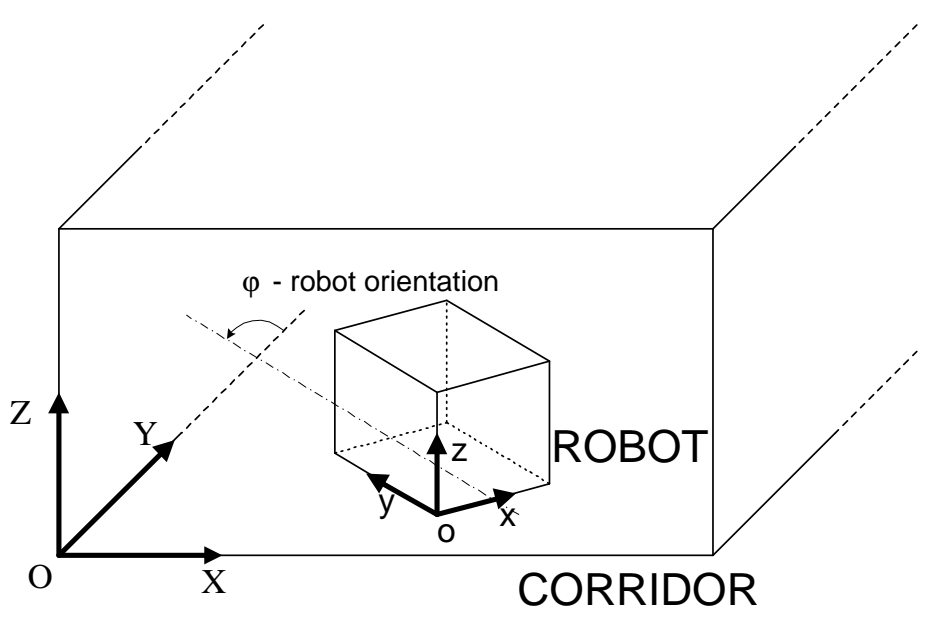

(a)

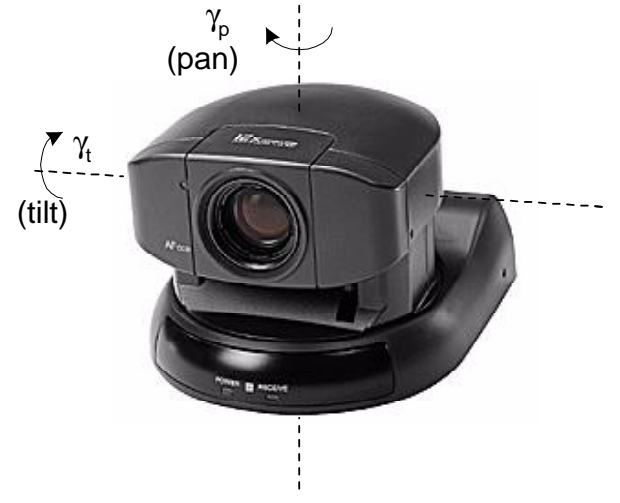

(b)

Figure 1:

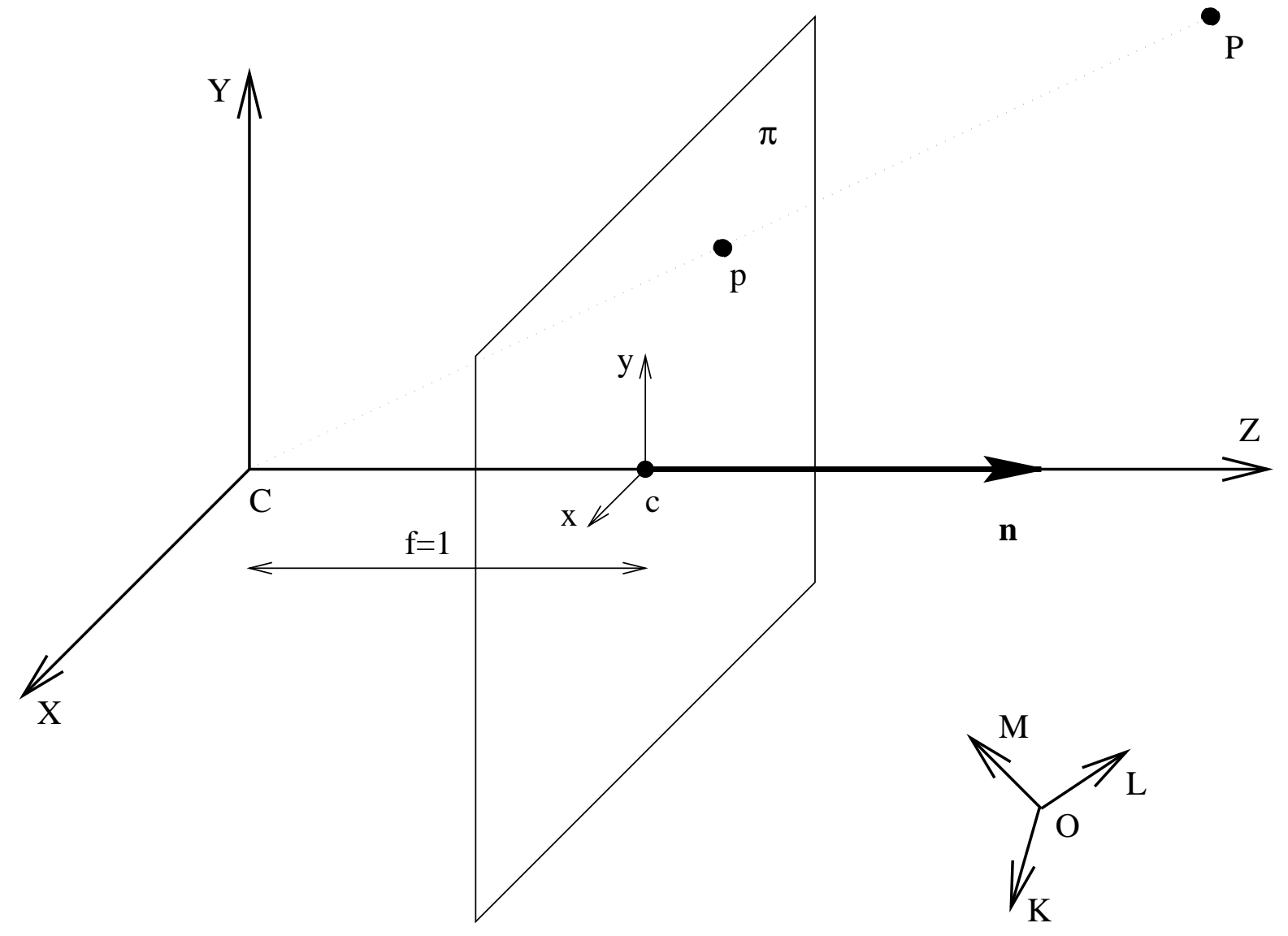

Figure 2: 


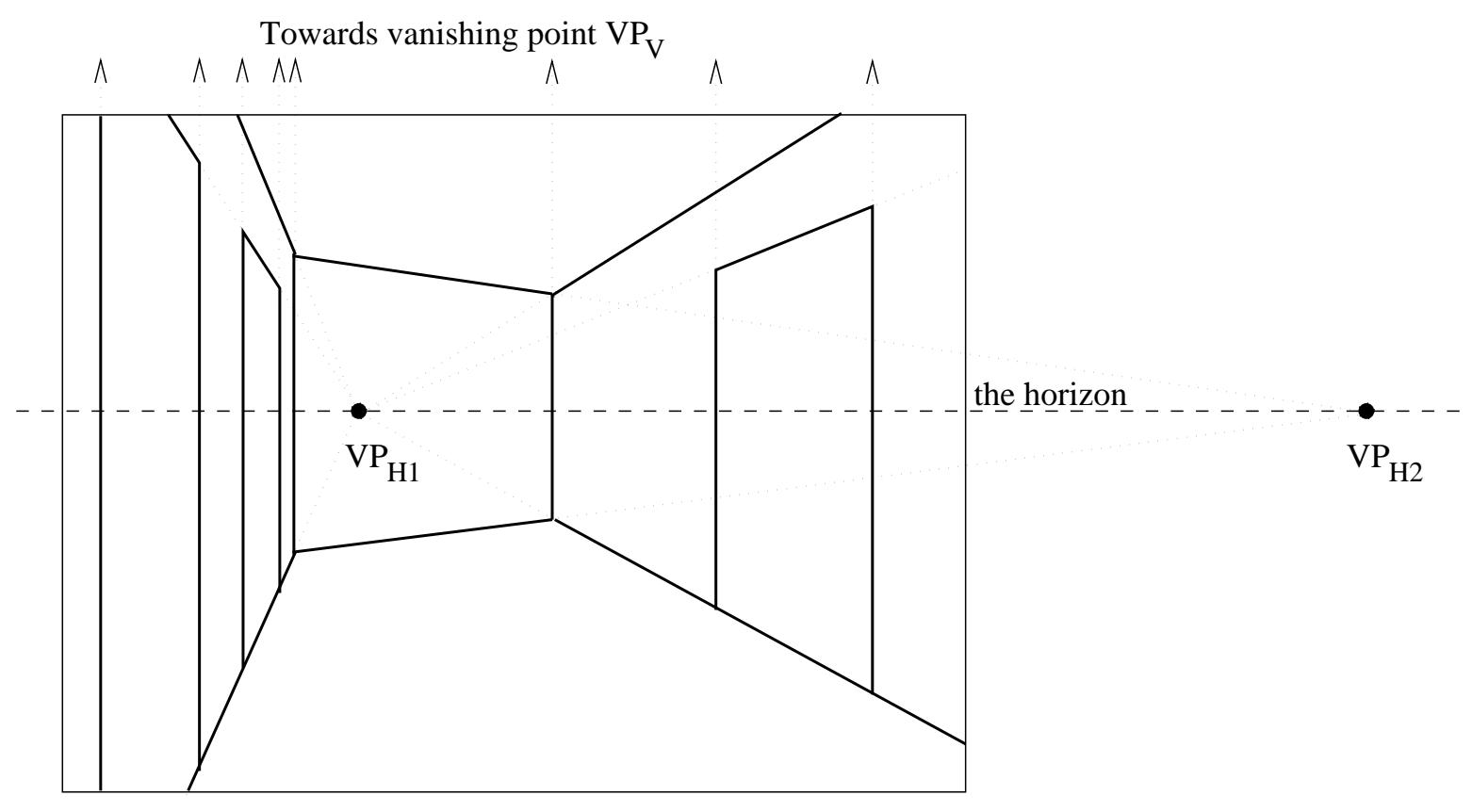

Figure 3:

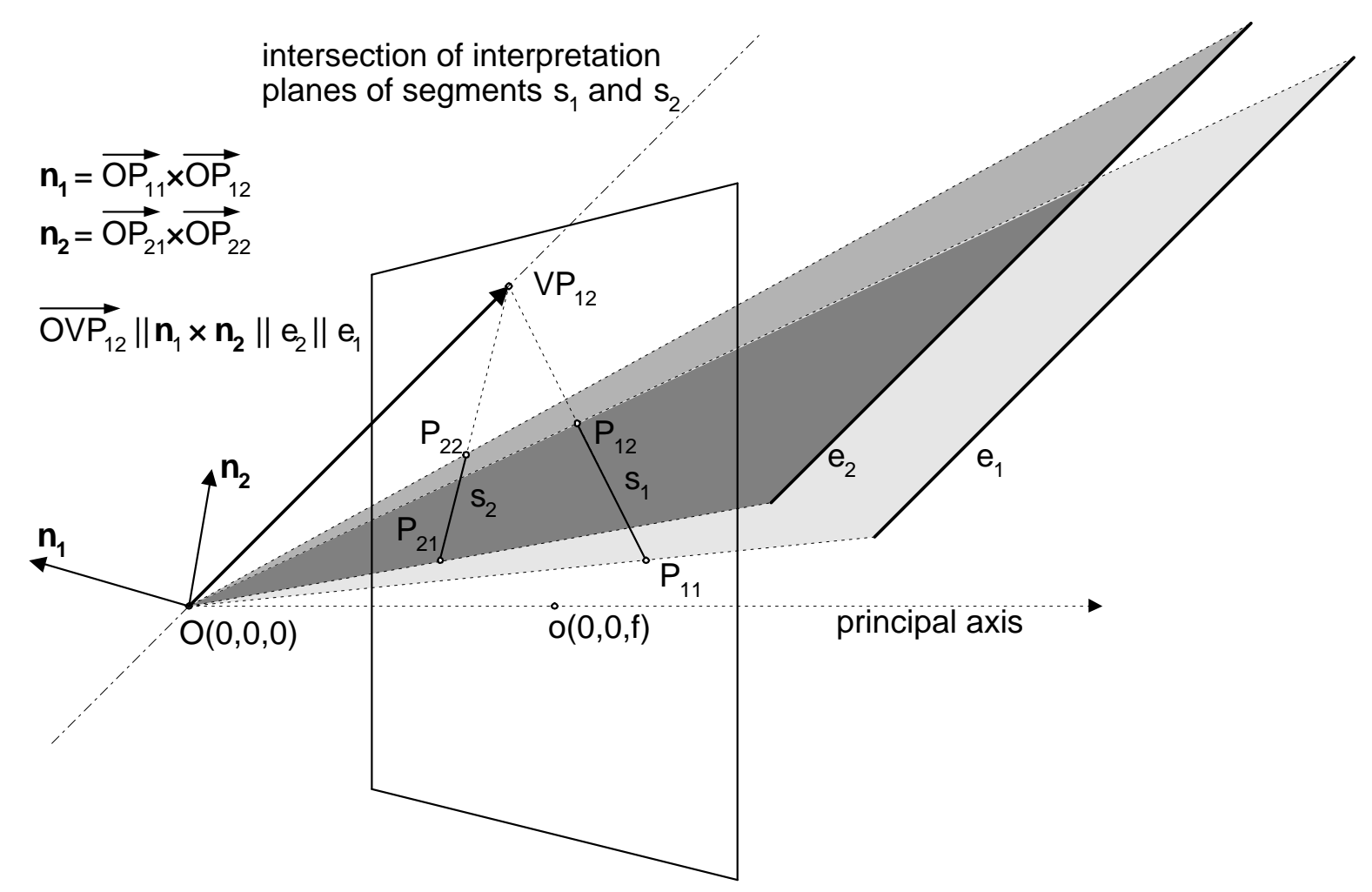

Figure 4: 


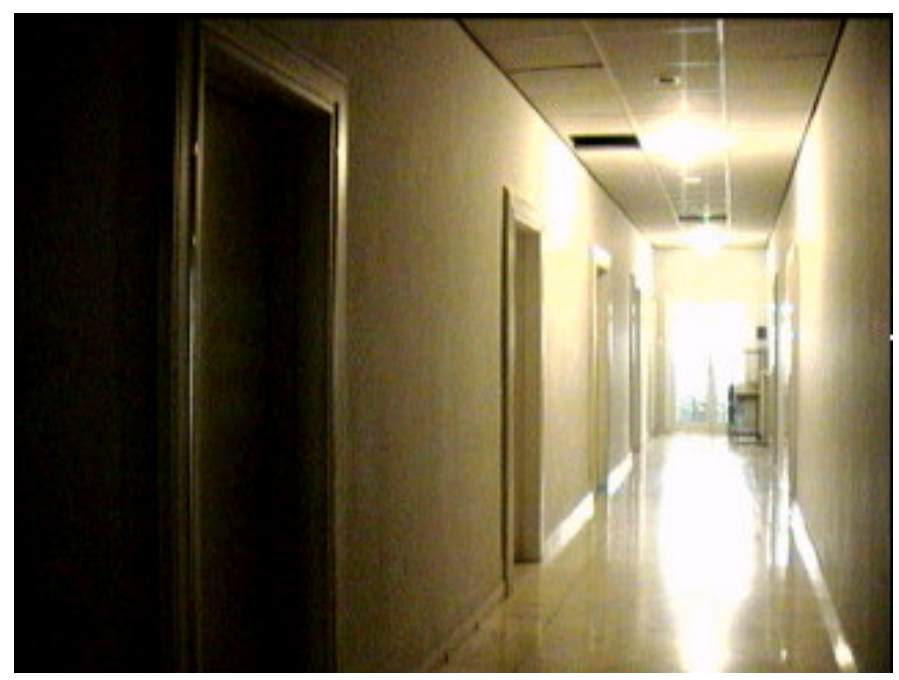

(a)

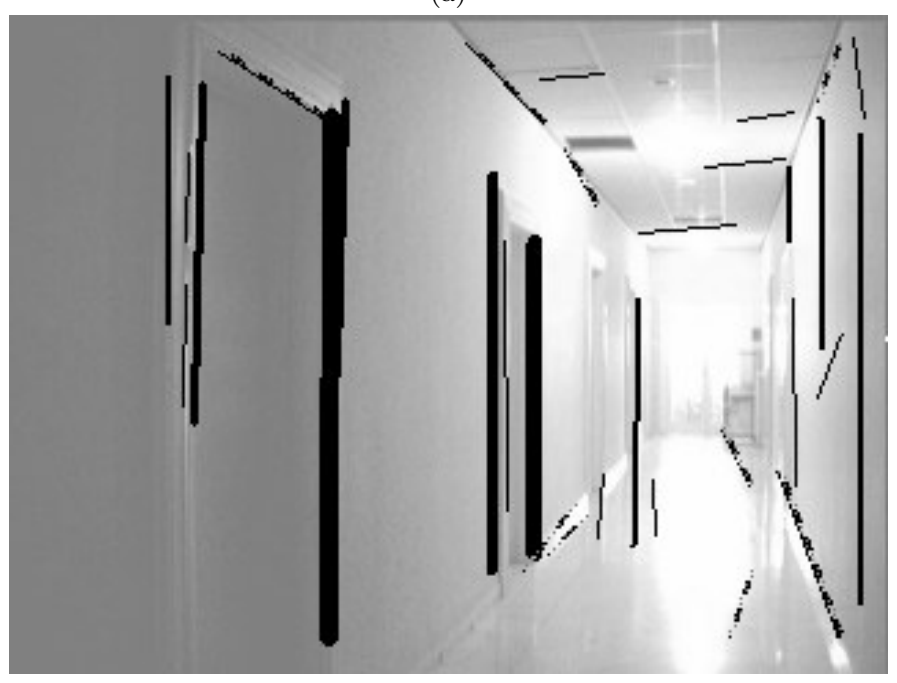

(b)

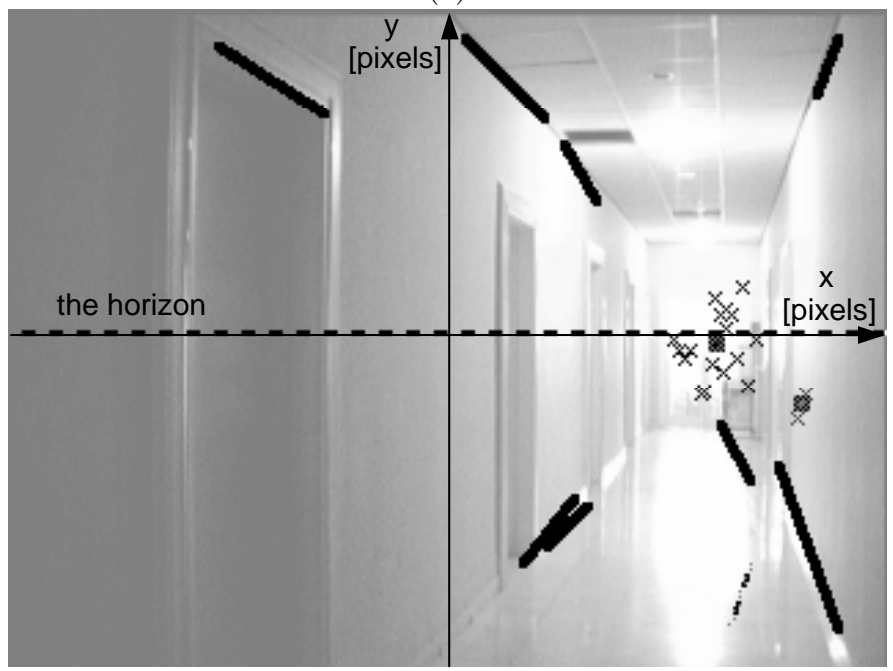

(c)

CAMERA: pan=0; tilt=0; hor'vew $=47$; ver'vew $=35$.

LINES: total $=31$; elected $=9$; support $=8$.

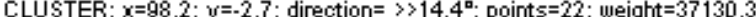

TIMING: smoothing $=243$; edges $=156$; lines $=250$; vanishing point $s=0$; total $=649 \mathrm{~ms}$.

(d)

Figure 5: 

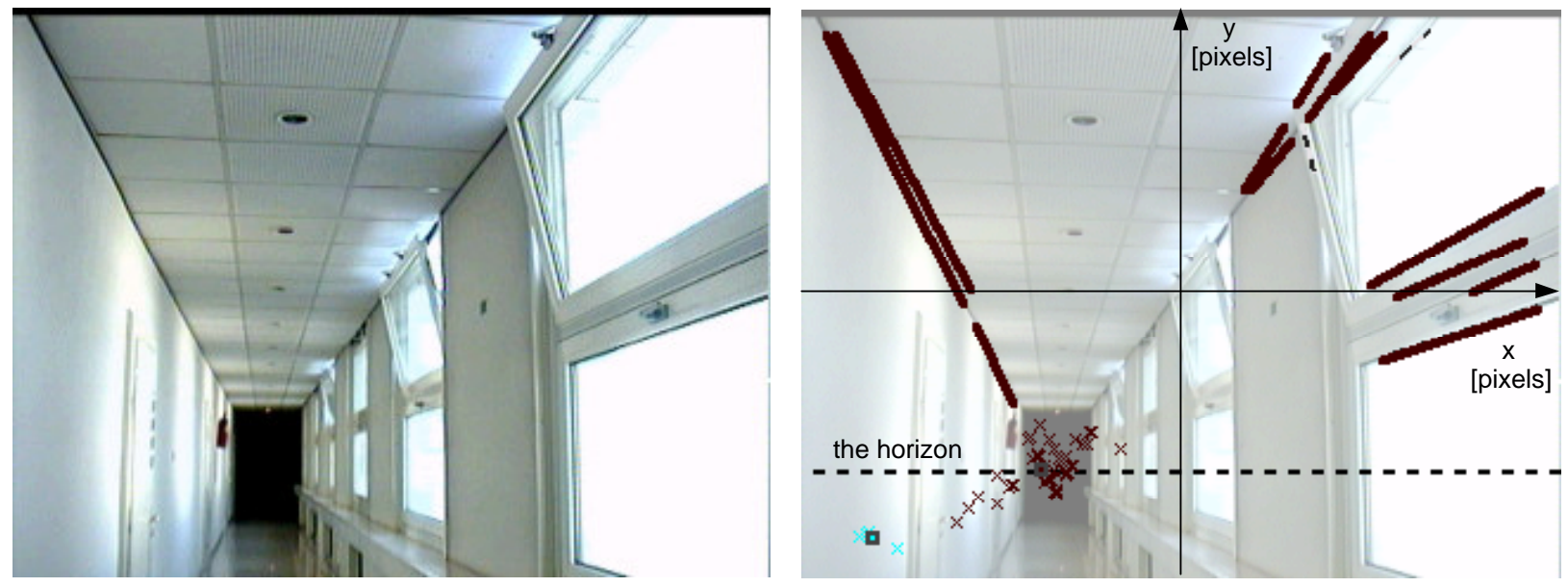

CAMERA: pan $=0$; tilt $=12$; hor'vew $=47$; ver'vew $=35$.

LINES: total $=40$; elected $=14 ;$ support $=12$.

CLUSTER: $x=-58.1, y=-74.1$; direction $=\left\langle<8.5^{\text {a }}\right.$; point $s=49$; weight $=315524.2$.

TIMING: smoothing $=258$; edges $=149$; lines $=203$; vanishing point $s=0 ;$ total $=610 \mathrm{~ms}$.

(a)
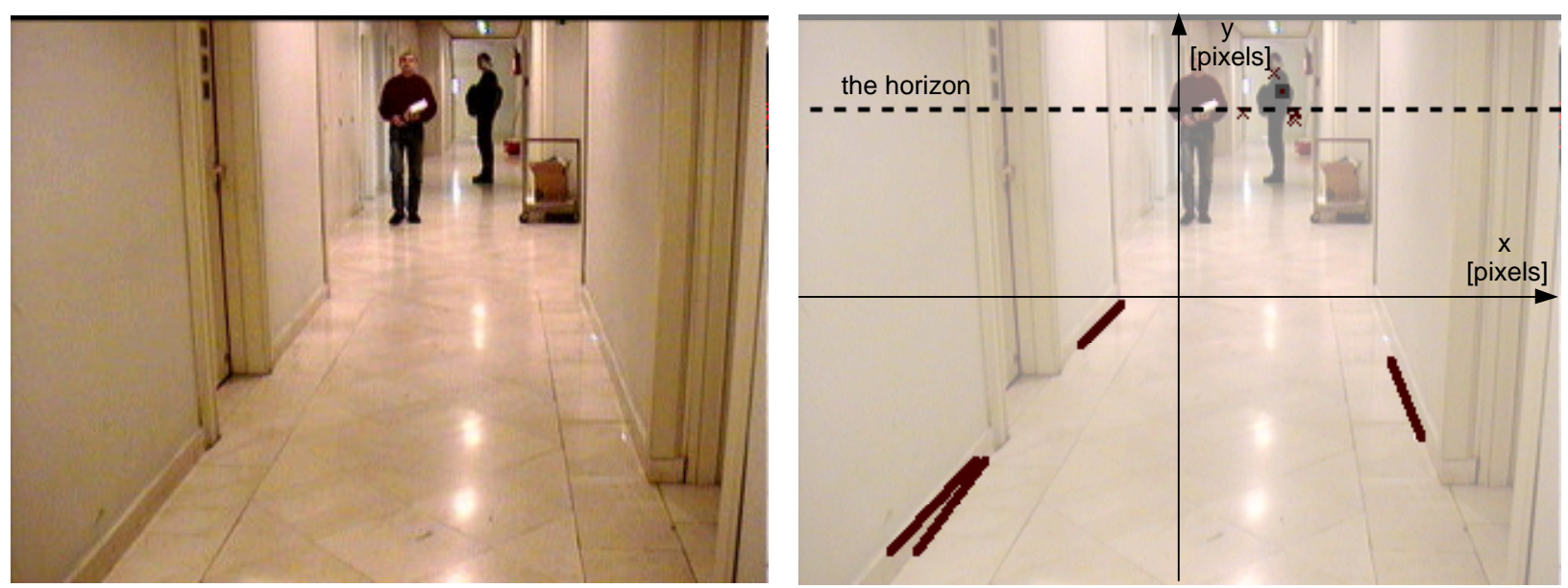

CAMERA: pan=0; tilt=-12; hor'vew $=47$; ver'wew $=35$.

LINES: total $=27 ;$ elected $=4 ;$ support $=4$.
CLUSTER: $x=43.4 ; y=87.3$; direction $=>>6.4^{\mathrm{a}} ;$ point $s=5 ;$ weight $=1522.5$

TIMING: smoothing $=258$; edges $=148$; lines $=195$; vanishing point $s=0$; total $=601 \mathrm{~ms}$

(b)

Figure 6: 

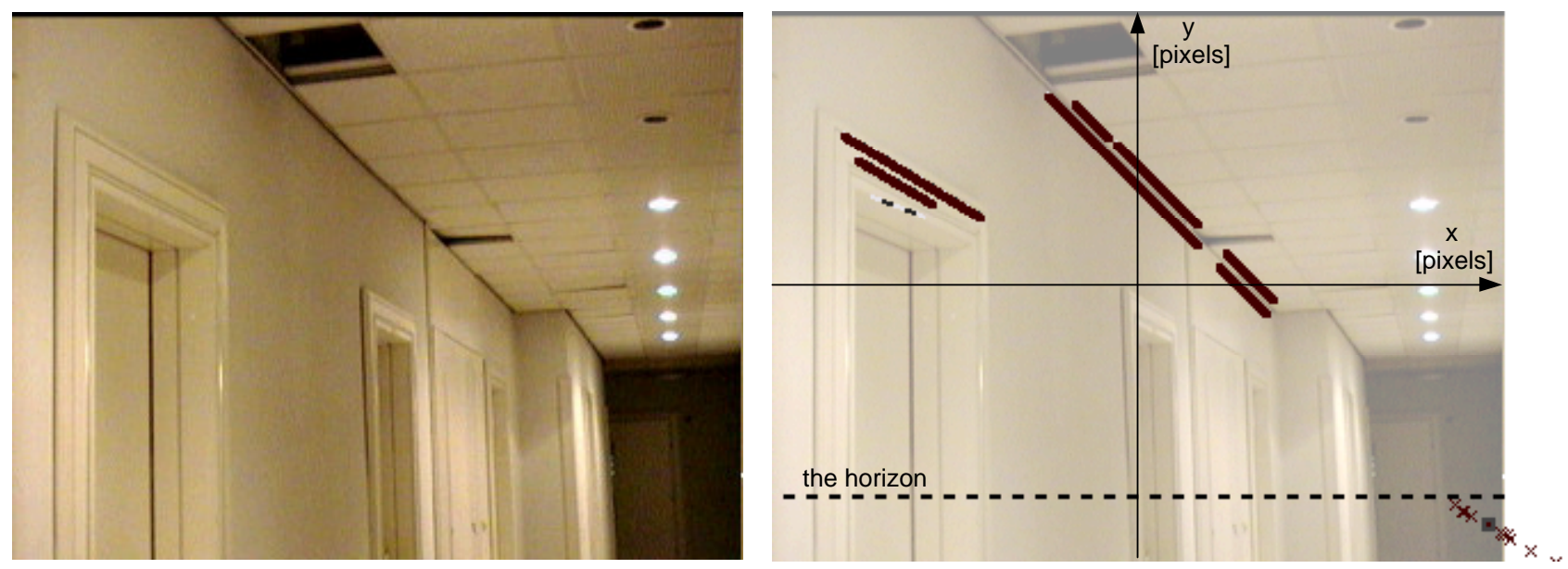

CAMERA: pan=0; tilt=10; hor'vew $=33$; ver'vew $=24$.

LINES: total=33; elected $=8$; support $=7$.

CLUSTER: $X=153.3 ; y=-104.6$; direction $=>15.8^{\mathrm{a}}$; point $s=11 ;$ weight $=6628.7$.

TIMING: smoothing $=258$; edges $=156$; lines $=180$; vanishing point $s=0 ;$ total $=594 \mathrm{~ms}$

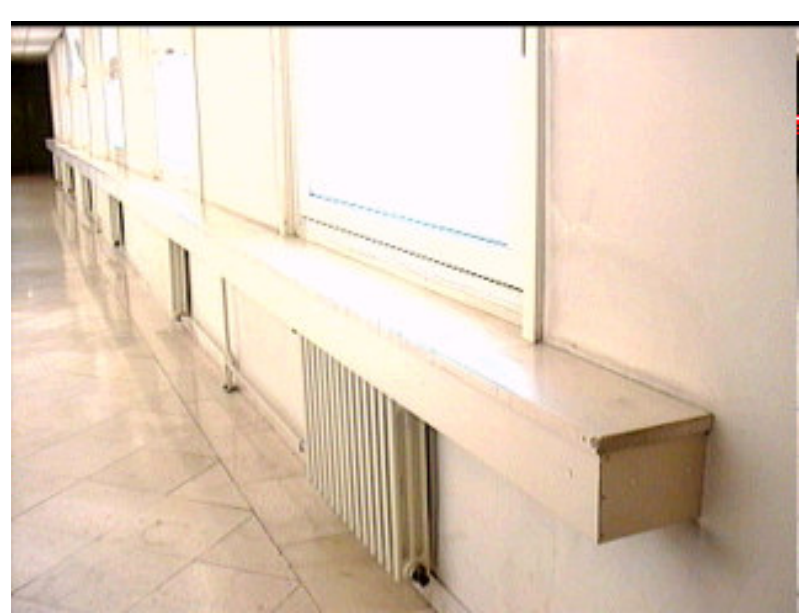

(a)

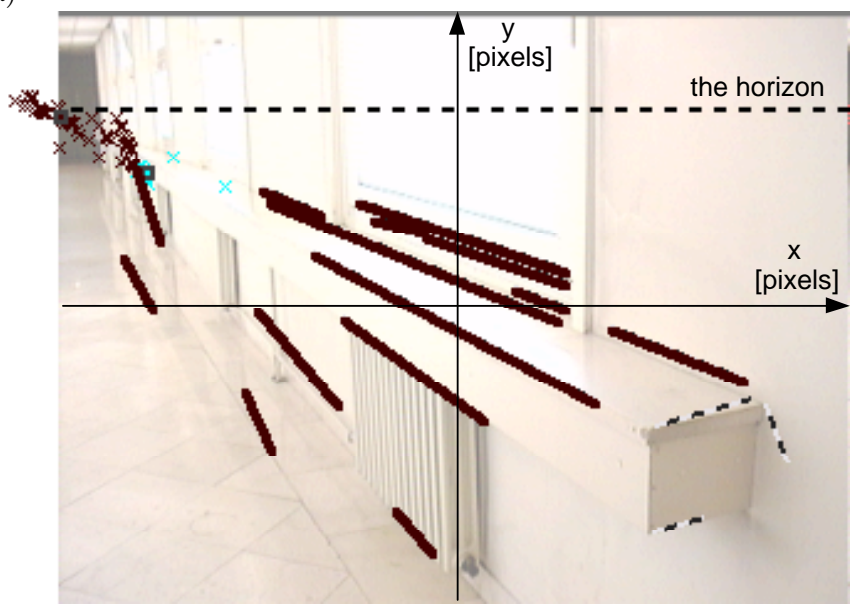

CAMERA: pan $=0$; tilt $=-12$; hor vew $=47$; ver'vew $=35$.

LINES: total $=31$; elected $=16$; support $=11$.

CLUSTER: $x=-165.7 ; y=79.2$, direction $=\left\langle 24.3^{\mathrm{a}}\right.$; point $s=36$; weight $=74659.2$ TIMUNG: smoothing=258; edges $=149$; lines $=171$; vanishing point $s=0$; total $=578 \mathrm{~ms}$

(b)

Figure 7: 


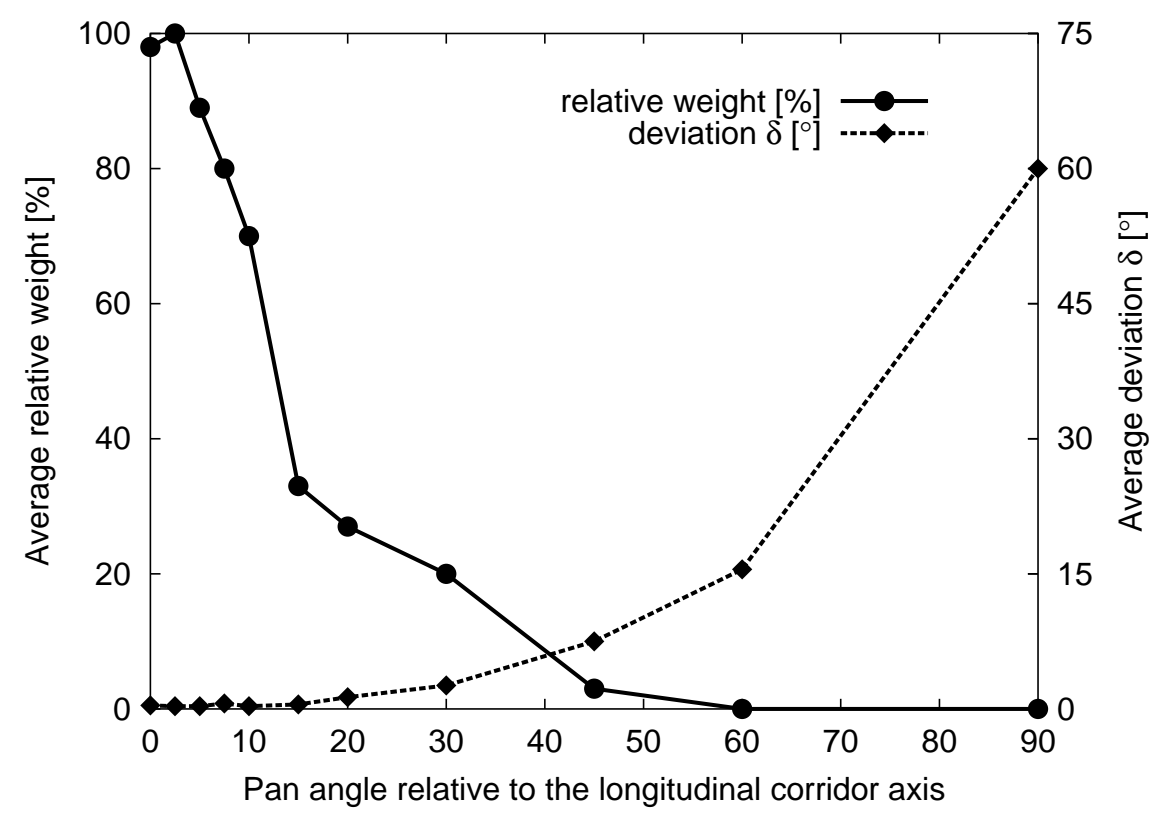

$\left(\gamma_{t}=15^{\circ}\right)$

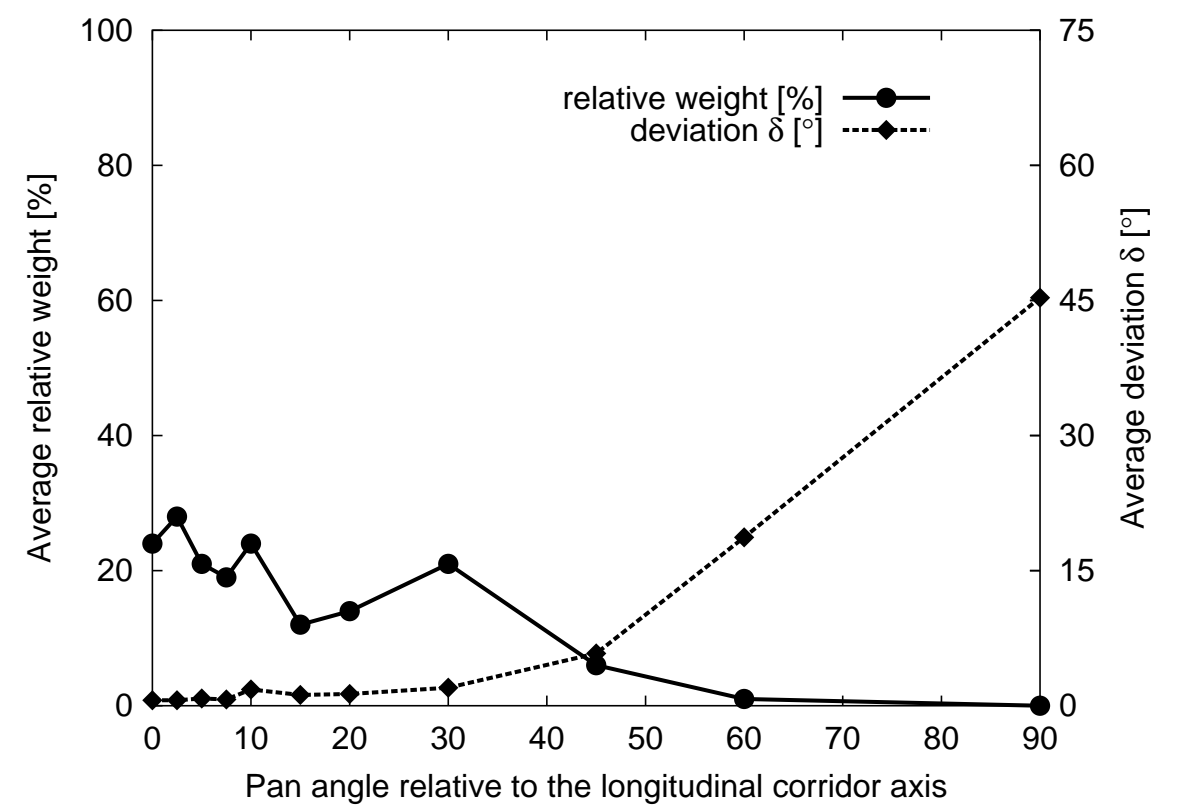

$\left(\gamma_{t}=-15^{\circ}\right)$

Figure 8: 


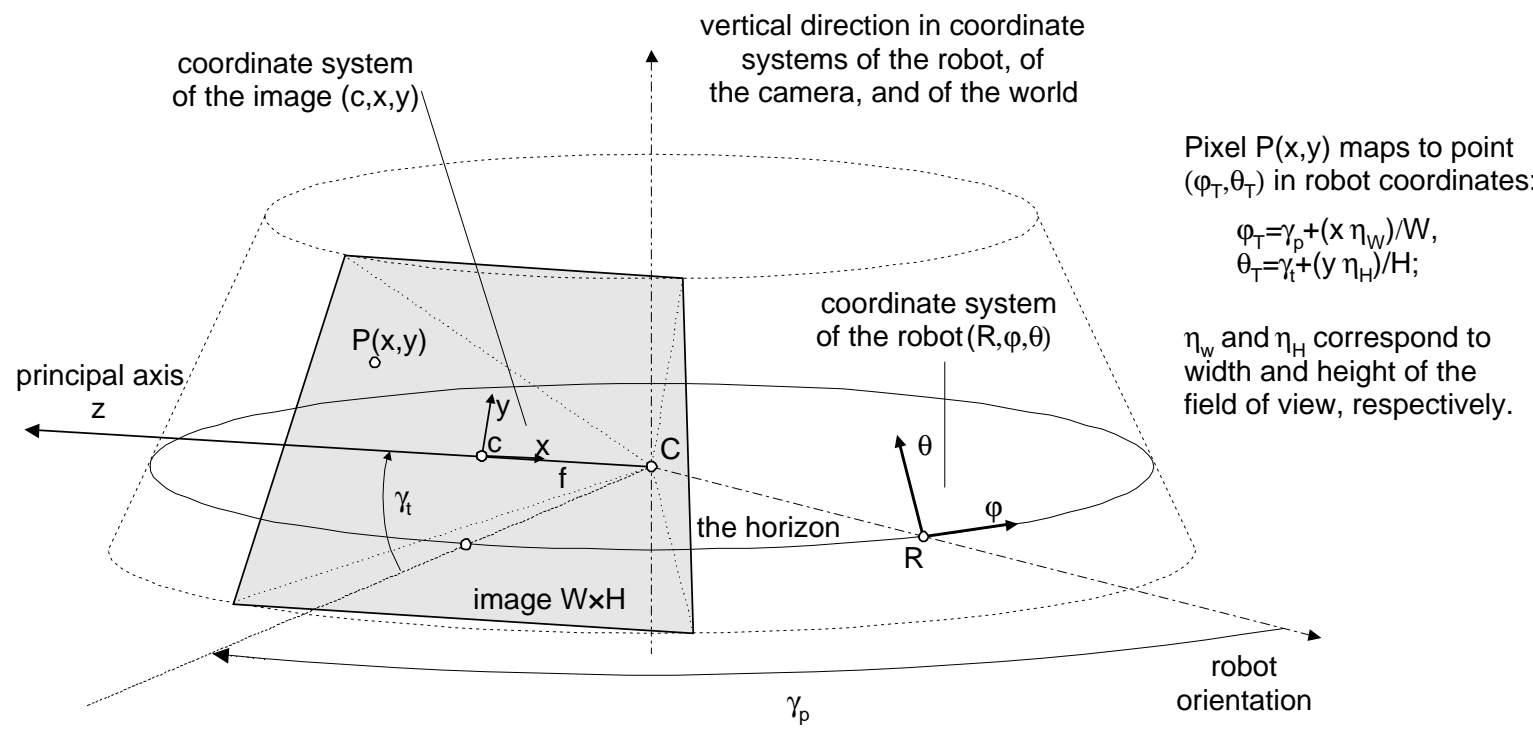

Figure 9:

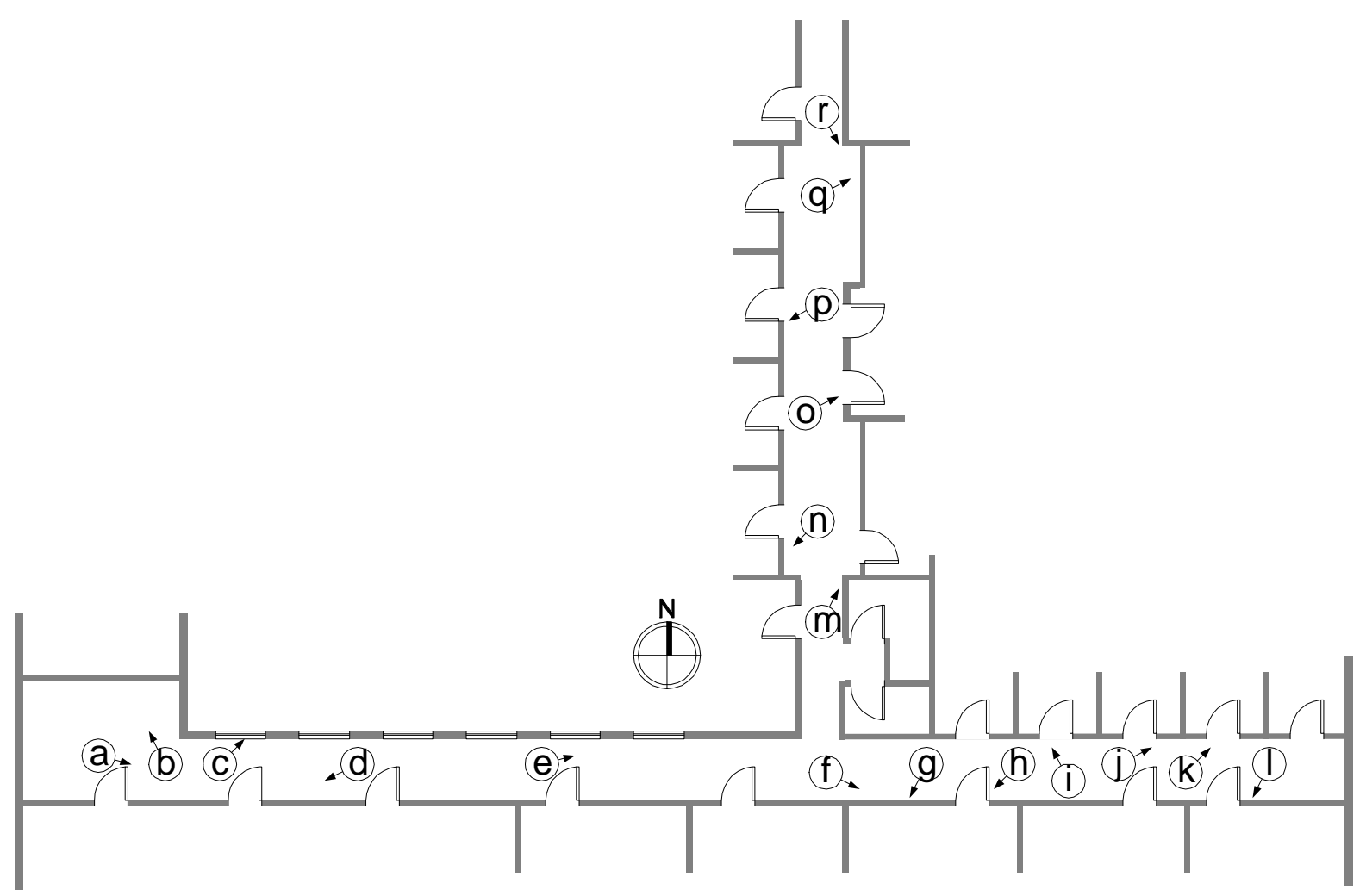

Figure 10: 

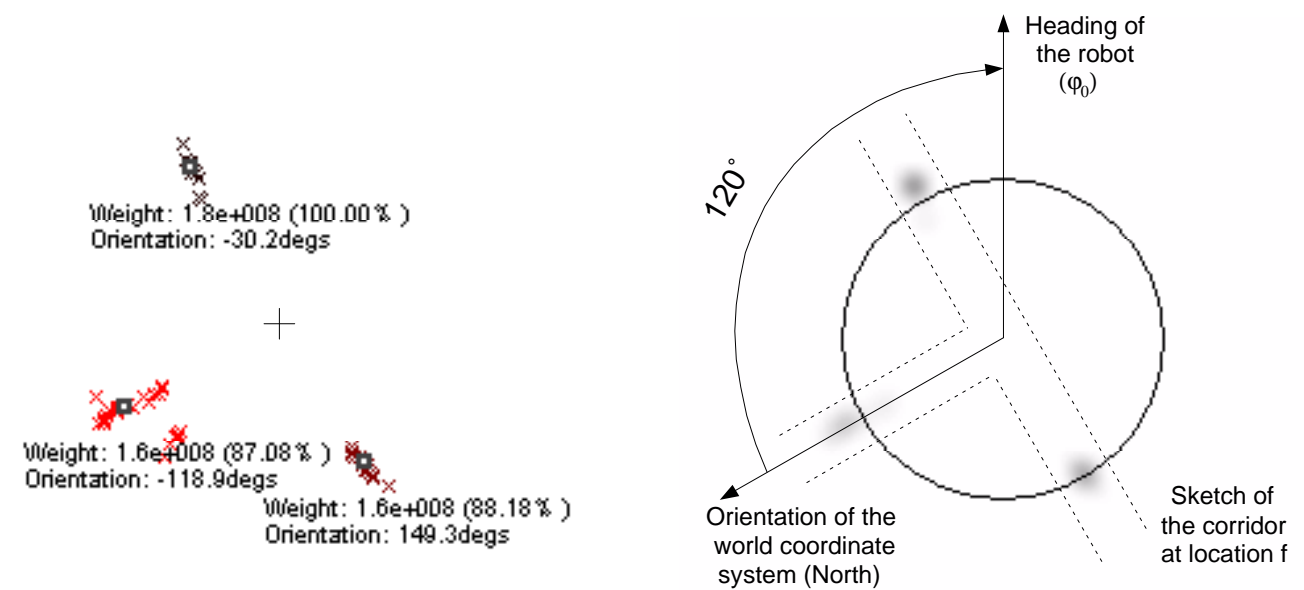

(a) Location $\mathbf{f}$

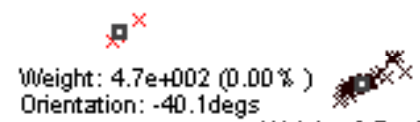

Orientation: -40 .1degs

$\times \quad+$ Uujeight: $2.7 e+008$ (100.00\%

ㄷ.

Uiveight: $1.6 \mathrm{e}+003(0.00 \%)$

Orientation: $-112.8 \mathrm{degs}$

viveight: 86 (0.00\%)

Orientation: 163.6 degs

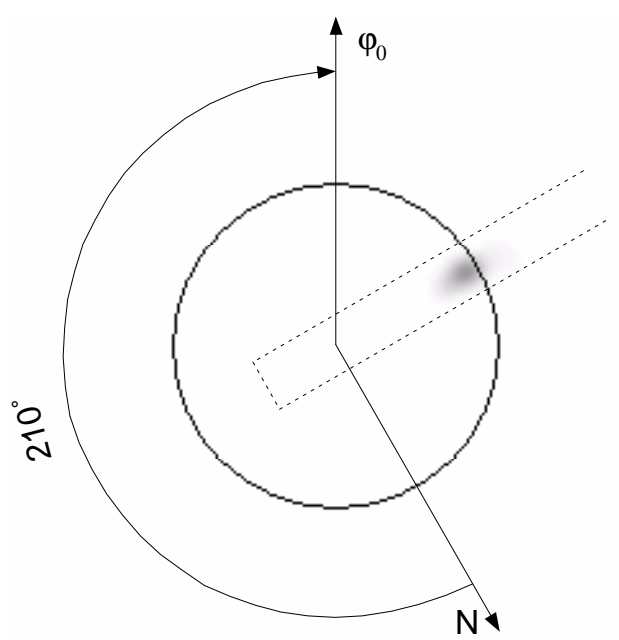

(b) Location 1

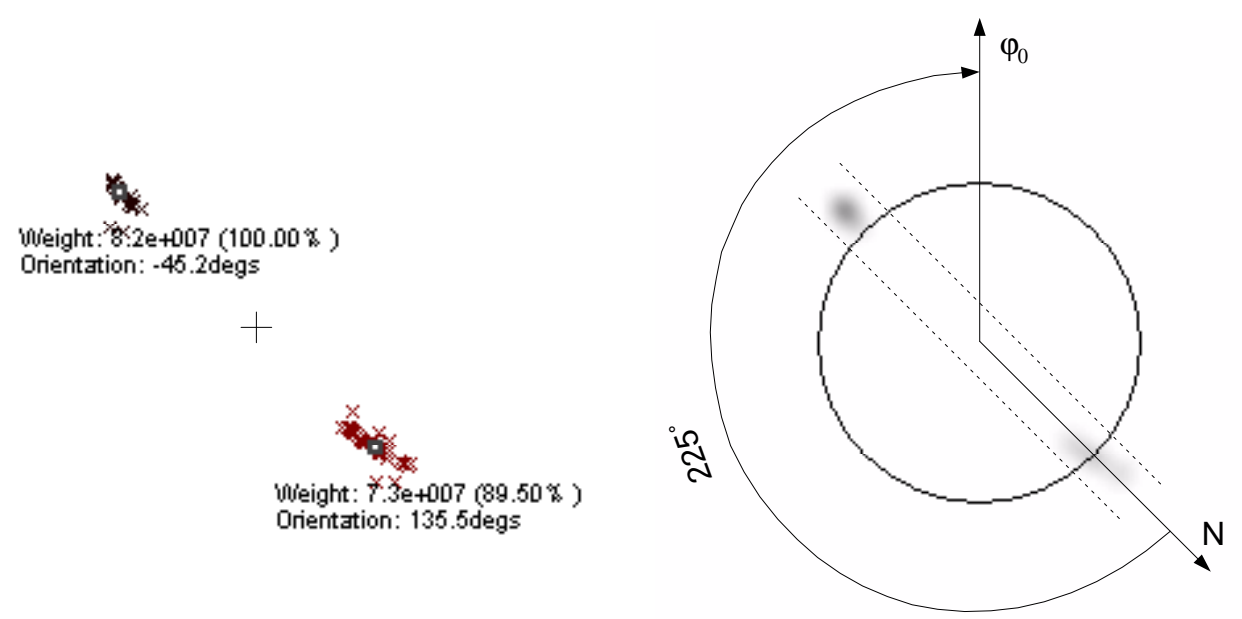

(c) Location $\mathbf{n}$

Figure 11: 
Table 1: The results of applying the method for the detection of vanishing points at six corridor locations (A, B, C, D, E, F) for different pan angles $\left(\gamma_{p}\right)$; tilt $\left(\gamma_{t}\right)$ angles of the camera were set to $15^{\circ}$ (left) and $-15^{\circ}$ (right).

\begin{tabular}{|c|c|c|c|c|c|c|}
\hline \multicolumn{2}{|c|}{$\gamma_{t}=15^{\circ}$} & \multicolumn{3}{|c|}{ count of line segments } & \multirow{2}{*}{ weight } & \multirow{2}{*}{$\delta$} \\
\hline$\gamma_{p}$ & $\#$ & total & elected & support & & \\
\hline \multirow{6}{*}{$0^{\circ}$} & $\overline{\mathrm{A}}$ & $\overline{266}$ & $\overline{99}$ & 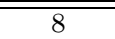 & 197166 & $0.1^{\circ}$ \\
\hline & B & 35 & 12 & 8 & 140814 & $0.0^{\circ}$ \\
\hline & $\mathrm{C}$ & 27 & 15 & 13 & 478203 & $0.3^{\circ}$ \\
\hline & $\mathrm{D}$ & 24 & 12 & 10 & 705075 & $1.2^{\circ}$ \\
\hline & $\mathrm{E}$ & 31 & 11 & 11 & 66937 & $0.4^{\circ}$ \\
\hline & $\mathrm{F}$ & 31 & 6 & 5 & 66681 & $0.4^{\circ}$ \\
\hline \multirow{6}{*}{$2.5^{\circ}$} & $\overline{\mathrm{A}}$ & 27 & 12 & 8 & 232618 & $0.5^{\circ}$ \\
\hline & $\mathrm{B}$ & 37 & 13 & 9 & 114607 & $0.3^{\circ}$ \\
\hline & $\mathrm{C}$ & 21 & 11 & 9 & 152993 & $0.1^{\circ}$ \\
\hline & $\mathrm{D}$ & 27 & 13 & 13 & 1038349 & $0.2^{\circ}$ \\
\hline & $\mathrm{E}$ & 36 & 11 & 11 & 85647 & $0.4^{\circ}$ \\
\hline & $\mathrm{F}$ & 30 & 6 & 6 & 56367 & $0.0^{\circ}$ \\
\hline \multirow{6}{*}{$5^{\circ}$} & $\bar{A}$ & 28 & 9 & 8 & 276781 & $0.4^{\circ}$ \\
\hline & B & 37 & 12 & 9 & 143783 & $0.4^{\circ}$ \\
\hline & $\mathrm{C}$ & 19 & 9 & 7 & 100972 & $0.0^{\circ}$ \\
\hline & $\mathrm{D}$ & 31 & 12 & 12 & 843077 & $0.2^{\circ}$ \\
\hline & $\mathrm{E}$ & 34 & 11 & 11 & 81498 & $0.4^{\circ}$ \\
\hline & $\mathrm{F}$ & 31 & 8 & 7 & 54430 & $0.6^{\circ}$ \\
\hline \multirow{6}{*}{$7.5^{\circ}$} & $\mathrm{A}$ & 34 & 12 & 10 & 265051 & $0.3^{\circ}$ \\
\hline & $\mathrm{B}$ & 41 & 14 & 11 & 151401 & $0.7^{\circ}$ \\
\hline & $\mathrm{C}$ & 19 & 10 & 8 & 74105 & $0.9^{\circ}$ \\
\hline & $\mathrm{D}$ & 31 & 15 & 14 & 700675 & $0.3^{\circ}$ \\
\hline & $\mathrm{E}$ & 32 & 11 & 11 & 114075 & $0.3^{\circ}$ \\
\hline & $\mathrm{F}$ & 34 & 11 & 10 & 44715 & $0.8^{\circ}$ \\
\hline \multirow{6}{*}{$10^{\circ}$} & $\overline{\mathrm{A}}$ & $\overline{31}$ & 13 & $\overline{11}$ & 137147 & $0.3^{\circ}$ \\
\hline & B & 32 & 11 & 7 & 139990 & $0.3^{\circ}$ \\
\hline & $\mathrm{C}$ & 19 & 11 & 10 & 116313 & $0.0^{\circ}$ \\
\hline & $\mathrm{D}$ & 31 & 15 & 14 & 617247 & $0.4^{\circ}$ \\
\hline & $\mathrm{E}$ & 32 & 11 & 10 & 141178 & $0.1^{\circ}$ \\
\hline & $\mathrm{F}$ & 31 & 8 & 8 & 26060 & $0.7^{\circ}$ \\
\hline \multirow{6}{*}{$15^{\circ}$} & $\mathrm{A}$ & 28 & 8 & $\overline{77}$ & 67169 & $0.1^{\circ}$ \\
\hline & B & 32 & 9 & 5 & 9313 & $1.0^{\circ}$ \\
\hline & $\mathrm{C}$ & 20 & 11 & 10 & 25156 & $0.4^{\circ}$ \\
\hline & $\mathrm{D}$ & 35 & 14 & 13 & 315271 & $0.0^{\circ}$ \\
\hline & $\mathrm{E}$ & 37 & 12 & 11 & 130775 & $1.2^{\circ}$ \\
\hline & $\mathrm{F}$ & 26 & 9 & 7 & 11352 & $0.2^{\circ}$ \\
\hline \multirow{6}{*}{$20^{\circ}$} & $\mathrm{A}$ & 28 & 11 & 7 & 20426 & $0.8^{\circ}$ \\
\hline & B & 27 & 8 & 7 & 33851 & $0.6^{\circ}$ \\
\hline & $\mathrm{C}$ & 11 & 7 & 4 & 18594 & $1.5^{\circ}$ \\
\hline & $\mathrm{D}$ & 35 & 14 & 13 & 315271 & $0.0^{\circ}$ \\
\hline & $\mathrm{E}$ & 39 & 12 & 9 & 60480 & $1.2^{\circ}$ \\
\hline & $\mathrm{F}$ & 22 & 5 & 4 & 6382 & $3.7^{\circ}$ \\
\hline \multirow{6}{*}{$30^{\circ}$} & $\overline{\bar{A}}$ & 26 & $\overline{88}$ & $\overline{5}$ & $\overline{c 10448}$ & $2.3^{\circ}$ \\
\hline & B & 18 & 6 & 4 & 22877 & $3.8^{\circ}$ \\
\hline & $\mathrm{C}$ & 21 & 13 & 3 & 15 & $1.8^{\circ}$ \\
\hline & $\mathrm{D}$ & 28 & 13 & 13 & 169710 & $0.6^{\circ}$ \\
\hline & $\mathrm{E}$ & 37 & 18 & 12 & 122139 & $2.3^{\circ}$ \\
\hline & $\mathrm{F}$ & 21 & 5 & 3 & 4071 & $4.7^{\circ}$ \\
\hline & $\overline{\mathrm{A}}$ & 15 & 6 & 4 & 376 & $2.0^{\circ}$ \\
\hline & B & 13 & 0 & - & - & - \\
\hline $45^{\circ}$ & $\mathrm{C}$ & 0 & - & - & - & - \\
\hline & $\mathrm{D}$ & 10 & 6 & 5 & 14421 & $6.4^{\circ}$ \\
\hline & $\mathrm{E}$ & 26 & 13 & 7 & 17535 & $6.4^{\circ}$ \\
\hline & $\mathrm{F}$ & 18 & 4 & 3 & 521 & $15^{\circ}$ \\
\hline & $\overline{\mathrm{A}}$ & 1 & 0 & - & - & - \\
\hline & B & 13 & 1 & 1 & 0 & $15^{\circ}$ \\
\hline $60^{\circ}$ & $\mathrm{C}$ & 0 & - & - & - & - \\
\hline & $\mathrm{D}$ & 0 & - & - & - & - \\
\hline & $\mathrm{E}$ & 4 & 0 & - & - & - \\
\hline & $\mathrm{F}$ & 11 & 1 & 1 & 0 & $16^{\circ}$ \\
\hline & $\mathrm{A}$ & 1 & 1 & $\overline{0}$ & $\overline{0}$ & $\overline{70^{\circ}}$ \\
\hline & B & 22 & 1 & 1 & 0 & $65^{\circ}$ \\
\hline $90^{\circ}$ & $\mathrm{C}$ & 0 & - & - & - & - \\
\hline & $\mathrm{D}$ & 21 & 0 & - & - & - \\
\hline & $\mathrm{E}$ & 1 & 1 & 1 & 0 & $45^{\circ}$ \\
\hline & $\mathrm{F}$ & 0 & - & - & - & - \\
\hline
\end{tabular}

\begin{tabular}{|c|c|c|c|c|c|c|}
\hline \multicolumn{2}{|c|}{$\gamma_{t}=-15^{\circ}$} & \multicolumn{3}{|c|}{ count of line segments } & \multirow{2}{*}{ weight } & \multirow{2}{*}{$\delta$} \\
\hline$\gamma_{p}$ & $\#$ & total & elected & support & & \\
\hline \multirow{6}{*}{$0^{\circ}$} & $\overline{\bar{A}}$ & 24 & 4 & 4 & 4205 & $0.3^{\circ}$ \\
\hline & B & 22 & 5 & 5 & 33789 & $0.8^{\circ}$ \\
\hline & $\mathrm{C}$ & 25 & 10 & 9 & 64671 & $0.4^{\circ}$ \\
\hline & $\mathrm{D}$ & 31 & 17 & 15 & 284802 & $0.3^{\circ}$ \\
\hline & $\mathrm{E}$ & 36 & 16 & 5 & 5665 & $0.9^{\circ}$ \\
\hline & $\mathrm{F}$ & 36 & 8 & 8 & 3390 & $0.9^{\circ}$ \\
\hline \multirow{6}{*}{$2.5^{\circ}$} & $\overline{\mathrm{A}}$ & 20 & 2 & 2 & 3146 & $0.5^{\circ}$ \\
\hline & B & 25 & 5 & 4 & 33295 & $0.5^{\circ}$ \\
\hline & $\mathrm{C}$ & 23 & 10 & 10 & 242975 & $0.5^{\circ}$ \\
\hline & $\mathrm{D}$ & 31 & 15 & 13 & 173212 & $0.0^{\circ}$ \\
\hline & $\mathrm{E}$ & 40 & 17 & 6 & 6428 & $0.8^{\circ}$ \\
\hline & $\mathrm{F}$ & 33 & 5 & 5 & 3763 & $1.5^{\circ}$ \\
\hline \multirow{6}{*}{$5^{\circ}$} & $\bar{A}$ & 27 & 4 & 4 & 3078 & $0.1^{\circ}$ \\
\hline & B & 22 & 6 & 5 & 41160 & $0.9^{\circ}$ \\
\hline & $\mathrm{C}$ & 23 & 10 & 10 & 242975 & $0.5^{\circ}$ \\
\hline & $\mathrm{D}$ & 29 & 13 & 10 & 55253 & $0.6^{\circ}$ \\
\hline & $\mathrm{E}$ & 32 & 16 & 7 & 5946 & $1.2^{\circ}$ \\
\hline & $\mathrm{F}$ & 28 & 5 & 5 & 3818 & $1.3^{\circ}$ \\
\hline \multirow{6}{*}{$7.5^{\circ}$} & $\mathrm{A}$ & 27 & 3 & 3 & 4659 & $0.3^{\circ}$ \\
\hline & B & 30 & 10 & 7 & 57488 & $0.8^{\circ}$ \\
\hline & $\mathrm{C}$ & 24 & 9 & 9 & 204531 & $0.8^{\circ}$ \\
\hline & $\mathrm{D}$ & 26 & 14 & 11 & 36593 & $0.1^{\circ}$ \\
\hline & $\mathrm{E}$ & 37 & 21 & 9 & 10024 & $1.0^{\circ}$ \\
\hline & $\mathrm{F}$ & 38 & 11 & 9 & 5982 & $1.0^{\circ}$ \\
\hline \multirow{6}{*}{$10^{\circ}$} & $\overline{\mathrm{A}}$ & $\overline{29}$ & $\overline{5}$ & 4 & 2666 & $1.2^{\circ}$ \\
\hline & B & 35 & 9 & 6 & 52800 & $0.5^{\circ}$ \\
\hline & $\mathrm{C}$ & 25 & 11 & 11 & 325675 & $0.6^{\circ}$ \\
\hline & $\mathrm{D}$ & 17 & 9 & 7 & 16694 & $1.5^{\circ}$ \\
\hline & $\mathrm{E}$ & 35 & 14 & 5 & 4920 & $1.7^{\circ}$ \\
\hline & $\mathrm{F}$ & 33 & 4 & 3 & 2010 & $5.3^{\circ}$ \\
\hline \multirow{6}{*}{$15^{\circ}$} & $\overline{\overline{\mathrm{A}}}$ & 25 & $\overline{3}$ & $\overline{2}$ & 3 & $1^{\circ}$ \\
\hline & B & 33 & 9 & 6 & 14178 & $1.8^{\circ}$ \\
\hline & C & 30 & 14 & 10 & 180777 & $1.6^{\circ}$ \\
\hline & $\mathrm{D}$ & 25 & 12 & 8 & 8056 & $1.6^{\circ}$ \\
\hline & $\mathrm{E}$ & 38 & 15 & 6 & 4076 & $0.3^{\circ}$ \\
\hline & $\mathrm{F}$ & 30 & 8 & 4 & 3 & $0.7^{\circ}$ \\
\hline \multirow{6}{*}{$20^{\circ}$} & $\bar{A}$ & 20 & 4 & 3 & 896 & $0.6^{\circ}$ \\
\hline & B & 26 & 6 & 4 & 14953 & $1.4^{\circ}$ \\
\hline & $\mathrm{C}$ & 26 & 12 & 10 & 210083 & $1.9^{\circ}$ \\
\hline & D & 21 & 12 & 4 & 2913 & $0.3^{\circ}$ \\
\hline & $\mathrm{E}$ & 35 & 15 & 7 & 2180 & $0.0^{\circ}$ \\
\hline & $\mathrm{F}$ & 32 & 8 & 5 & 278 & $3.4^{\circ}$ \\
\hline \multirow{6}{*}{$30^{\circ}$} & $\bar{A}$ & 25 & 4 & 4 & 997 & $0.5^{\circ}$ \\
\hline & B & 14 & 3 & 3 & 2 & $3.9^{\circ}$ \\
\hline & $\mathrm{C}$ & 30 & 18 & 16 & 344173 & $0.4^{\circ}$ \\
\hline & $\mathrm{D}$ & 14 & 9 & 8 & 1398 & $2.8^{\circ}$ \\
\hline & $\mathrm{E}$ & 32 & 9 & 4 & 662 & $3.3^{\circ}$ \\
\hline & $\mathrm{F}$ & 24 & 2 & 1 & 1 & $0.9^{\circ}$ \\
\hline & $\overline{\overline{\mathrm{A}}}$ & 13 & 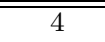 & 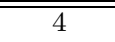 & 199 & $7.9^{\circ}$ \\
\hline & B & 13 & 3 & 2 & 1 & $2.2^{\circ}$ \\
\hline $45^{\circ}$ & $\mathrm{C}$ & 23 & 16 & 12 & 88651 & $6.2^{\circ}$ \\
\hline & $\mathrm{D}$ & 1 & 1 & 1 & 0 & $3.1^{\circ}$ \\
\hline & $\mathrm{E}$ & 35 & 11 & 4 & 4090 & $8.6^{\circ}$ \\
\hline & $\mathrm{F}$ & 28 & 6 & 5 & 2 & $6.9^{\circ}$ \\
\hline & $\bar{A}$ & 0 & - & - & - & - \\
\hline & B & 1 & 1 & 1 & 0 & $9^{\circ}$ \\
\hline $60^{\circ}$ & $\mathrm{C}$ & 17 & 12 & 6 & 7918 & $19^{\circ}$ \\
\hline & $\mathrm{D}$ & 0 & - & - & - & - \\
\hline & $\mathrm{E}$ & 21 & 3 & 3 & 4 & $28^{\circ}$ \\
\hline & $\mathrm{F}$ & 14 & 0 & - & - & - \\
\hline & $\overline{\mathrm{A}}$ & 19 & 4 & 3 & $\overline{\overline{2}}$ & $63^{\circ}$ \\
\hline & B & 1 & 1 & 1 & 0 & $7^{\circ}$ \\
\hline $90^{\circ}$ & $\mathrm{C}$ & 26 & 0 & - & - & - \\
\hline & D & 0 & - & - & - & - \\
\hline & $\mathrm{E}$ & 11 & 1 & 1 & 0 & $66^{\circ}$ \\
\hline & $\mathrm{F}$ & 6 & 0 & - & - & - \\
\hline
\end{tabular}


Table 2: The results of applying the method for the detection of vanishing points to sets of pseudorandom line segments.

\begin{tabular}{|c|c||c|c|c||c|c|}
\hline \multirow{2}{*}{ series } & \multirow{2}{*}{ count } & \multicolumn{3}{c||}{ average count of segments } & \multicolumn{2}{c|}{ ratio $\frac{\text { support }}{\text { elected }}$} \\
\cline { 3 - 7 } & & total & elected & support & $\mu$ & $\sigma$ \\
\hline A & 20 & 20 & 7.4 & 3.1 & 0.38 & 0.18 \\
\hline B & 20 & 40 & 13.7 & 6.1 & 0.45 & 0.09 \\
\hline C & 20 & 60 & 19.9 & 8.5 & 0.42 & 0.10 \\
\hline
\end{tabular}


Table 3: The results of applying the localization procedure at locations designated in Fig.10.

\begin{tabular}{|c|c|c|c|c|}
\hline \multirow{2}{*}{ location } & \multirow{2}{*}{$\varphi_{0}\left[^{\circ}\right]$} & \multicolumn{3}{|c|}{ clusters } \\
\hline & & $\varphi\left[^{\circ}\right]$ & wt [\%] & $\delta$ \\
\hline \multirow[b]{2}{*}{$\mathrm{a}$} & \multirow{2}{*}{120} & -30.0 & 100 & 0.0 \\
\hline & & 150.1 & 4 & 0.1 \\
\hline \multirow{2}{*}{ b } & \multirow{2}{*}{330} & 121.4 & 100 & 1.4 \\
\hline & & -59.5 & 17 & 0.5 \\
\hline \multirow{2}{*}{ c } & \multirow{2}{*}{45} & 47.8 & 100 & 2.8 \\
\hline & & -133.9 & 21 & 1.1 \\
\hline \multirow{2}{*}{ d } & \multirow{2}{*}{240} & 30.2 & 100 & 0.2 \\
\hline & & -150.0 & 75 & 0.0 \\
\hline \multirow{2}{*}{$\mathrm{e}$} & \multirow{2}{*}{60} & 29.6 & 100 & 0.4 \\
\hline & & -149.8 & 27 & 0.2 \\
\hline \multirow{3}{*}{$\mathrm{f}$} & \multirow{3}{*}{120} & -30.2 & 100 & 0.2 \\
\hline & & 149.3 & 88 & 0.7 \\
\hline & & -118.9 & 87 & 1.1 \\
\hline \multirow{2}{*}{ g } & \multirow{2}{*}{210} & -120.3 & 100 & 0.3 \\
\hline & & 60.9 & 5 & 0.9 \\
\hline \multirow{2}{*}{ h } & \multirow{2}{*}{225} & -133.3 & 100 & 1.7 \\
\hline & & 44.6 & 17 & 0.4 \\
\hline \multirow{2}{*}{$\mathrm{i}$} & \multirow{2}{*}{330} & 120.2 & 100 & 0.2 \\
\hline & & -60.2 & 80 & 0.2 \\
\hline \multirow{2}{*}{$\mathrm{j}$} & \multirow{2}{*}{60} & -149.2 & 100 & 0.8 \\
\hline & & 30.4 & 38 & 0.4 \\
\hline \multirow{2}{*}{$\mathrm{k}$} & \multirow{2}{*}{45} & -135.3 & 100 & 0.3 \\
\hline & & 45.1 & 70 & 0.1 \\
\hline l & 210 & 60.6 & 100 & 0.6 \\
\hline \multirow{2}{*}{$\mathrm{m}$} & \multirow{2}{*}{30} & -30.7 & 100 & 0.7 \\
\hline & & 150.4 & 0.01 & 0.4 \\
\hline \multirow{2}{*}{$\mathrm{n}$} & 225 & -45.2 & 100 & 0.2 \\
\hline & & 135.5 & 90 & 0.5 \\
\hline 0 & 60 & 119.7 & 100 & 0.3 \\
\hline 0 & & -59.9 & 63 & 0.1 \\
\hline $\mathrm{D}$ & 240 & 120.9 & 100 & 0.9 \\
\hline$P$ & & -59.8 & 7 & 0.2 \\
\hline O & 60 & -60.0 & 100 & 0.0 \\
\hline 4 & 00 & 119.1 & 26 & 0.9 \\
\hline$r$ & 150 & 30.7 & 100 & 0.7 \\
\hline 1 & & -150.5 & 57 & 0.5 \\
\hline
\end{tabular}

